Effects of WENO flux reconstruction order and spatial resolution on reshocked two-dimensional Richtmyer-Meshkov instability

M. Latini, O. Schilling, W. Don

March 22, 2006 
This document was prepared as an account of work sponsored by an agency of the United States Government. Neither the United States Government nor the University of California nor any of their employees, makes any warranty, express or implied, or assumes any legal liability or responsibility for the accuracy, completeness, or usefulness of any information, apparatus, product, or process disclosed, or represents that its use would not infringe privately owned rights. Reference herein to any specific commercial product, process, or service by trade name, trademark, manufacturer, or otherwise, does not necessarily constitute or imply its endorsement, recommendation, or favoring by the United States Government or the University of California. The views and opinions of authors expressed herein do not necessarily state or reflect those of the United States Government or the University of California, and shall not be used for advertising or product endorsement purposes.

This work was performed under the auspices of the U.S. Department of Energy by University of California, Lawrence Livermore National Laboratory under Contract W-7405-Eng-48. 


\title{
Effects of WENO flux reconstruction order and spatial resolution on reshocked two-dimensional Richtmyer-Meshkov instability
}

\author{
Marco Latini ${ }^{a}$ Oleg Schilling ${ }^{\mathrm{b}, *}$ Wai Sun Don ${ }^{\mathrm{c}}$ \\ ${ }^{a}$ Applied and Computational Mathematics, California Institute of Technology, \\ Pasadena, California 91125 \\ ${ }^{\mathrm{b}}$ University of California, Lawrence Livermore National Laboratory, \\ Livermore, California 94551 \\ ${ }^{\mathrm{c}}$ Division of Applied Mathematics, Brown University, \\ Providence, Rhode Island 02912
}

\begin{abstract}
Finite-difference weighted essentially non-oscillatory (WENO) simulations of the reshocked two-dimensional single-mode Richtmyer-Meshkov instability using third-, fifth- and ninth-order spatial flux reconstruction and uniform spatial grid resolutions corresponding to 128,256 and 512 points per initial perturbation wavelength are presented. The dependence of the density, vorticity, simulated density Schlieren and baroclinic production fields, mixing layer width, circulation deposition, mixing profiles, chemical products and mixing fractions, energy spectra, statistics, probability distribution functions, effective turbulent kinetic energy and enstrophy production/dissipation rates, numerical Reynolds numbers, and effective numerical viscosity on the order and resolution is comprehensively investigated to long evolution times. The results are interpreted using the computed implicit numerical diffusion arising from the truncation errors in the characteristic projection-based WENO method. It is quantitatively shown that simulations with higher order and higher resolution have lower numerical dissipation. The sensitivity of the quantities considered to the order and resolution is further amplified following reshock, when the energy deposition on the evolving interface by the second shock-interface interaction induces the formation of small-scale structures. Simulations using lower orders of reconstruction and on coarser grids preserve large-scale structures and flow symmetry to late times, while simulations using higher orders of reconstruction and on finer grids exhibit fragmentation of the structures, symmetry breaking and increased mixing. The investigation demonstrates that similar flow features are qualitatively and quantitatively captured by either approximately doubling the order or the resolution. Additionally, the computational scaling shows that increasing the order is more
\end{abstract}


advantageous than doubling the resolution for the complex shock-driven hydrodynamic flow and WENO method considered here. The present investigation suggests that the ninth-order WENO method is well-suited for the simulation and analysis of complex multi-scale flows and mixing generated by shock-induced hydrodynamic instabilities.

Key words: Richtmyer-Meshkov instability, weighted essentially non-oscillatory (WENO) method, mixing properties, reshock, numerical dissipation

\section{Introduction}

The Richtmyer-Meshkov instability is a fundamental fluid instability that develops when perturbations on an interface separating gases with different properties grow following the passage of a shock. This instability is typically studied in shock tube experiments, in which an incident shock passes through an initially perturbed interface separating the gases. Following the passage of the shock, the interface is set in motion along the direction of shock propagation and a transmitted shock enters the second gas. The misalignment of the density and pressure gradients cause a deposition of vorticity $\boldsymbol{\omega} \equiv \boldsymbol{\nabla} \times \boldsymbol{u}$ on the interface through baroclinic vorticity production. In two dimensions, the vorticity equation is

$$
\frac{\mathrm{d} \boldsymbol{\omega}}{\mathrm{d} t}=\mathcal{P}-\boldsymbol{\omega} \nabla \cdot \boldsymbol{u}
$$

where $\mathrm{d} / \mathrm{d} t=\partial / \partial t+\mathbf{u} \cdot \nabla$ is the convective derivative and $\mathcal{P} \equiv(\nabla \rho \times \nabla p) / \rho^{2}$ is the baroclinic vorticity production term. The vorticity deposited by the shock on the interface drives the instability, which results in interpenetrating bubbles and spikes. At later times, complex roll-up structures form on the spike and the vorticity forms strong cores. The transmitted shock reflects from the end wall of the shock tube and interacts with the evolving interface during the reshock phase, further contributing to the development of complex interacting fluid and wave structures.

In the present work, the formally high-order accurate weighted essentially non-oscillatory (WENO) shock-capturing method using a third-order totalvariation diminishing (TVD) Runge-Kutta time-evolution scheme ([1] and references therein) is applied to the two-dimensional single-mode RichtmyerMeshkov instability with reshock for long evolution times. The initial conditions and computational domain for the simulations are modeled [2] after the

\footnotetext{
* Corresponding author.

Email addresses: mlatini@acm.caltech.edu (Marco Latini), schilling1@llnl.gov (Oleg Schilling), wsdon@cfm.brown.edu (Wai Sun Don).
} 
single-mode, Mach 1.21 air(acetone)/ $\mathrm{SF}_{6}$ shock tube experiment of Collins and Jacobs [3]. As the Richtmyer-Meshkov instability-induced flow contains shock waves, a direct numerical simulation (DNS) is not possible due to the prohibitively small scales needed to resolve the complex interactions of the shock with the density interfaces and the shocks themselves. As a result, numerical investigations of the Richtmyer-Meshkov instability typically utilize conservative Eulerian shock-capturing methods that do not resolve all of the spatial scales and small-scale interactions, but instead ensure that fundamental quantities are conserved across a shock and that the shock speed is accurately captured. As numerical results from such simulations are used to interpret and to better understand the physical mechanisms in the dynamical evolution of the Richtmyer-Meshkov instability, it is essential to ascertain how such results depend on the numerical algorithm (e.g., the order of WENO flux reconstruction and the resolution in the present study).

The objective of this study is to self-consistently and systematically explore and quantify the sensitivity of a broad array of quantities characterizing singlemode Richtmyer-Meshkov instability-induced mixing on the order of WENO flux reconstruction (third, fifth and ninth) and on the grid resolution $(128,256$ and 512 points per initial perturbation wavelength $\lambda$ ). Many of these quantities were previously considered in the description of amplitude growth [2] and of the physics of reshock [4]. In the present work, the density, vorticity, simulated density Schlieren and baroclinic vorticity production fields are qualitatively compared from the simulations. Also compared quantitatively are the mixing layer widths, circulation, mixing profiles, chemical products and mixing fractions, energy spectra, statistics, probability distribution functions, effective turbulent kinetic energy and enstrophy production/dissipation rates, numerical Reynolds numbers and effective numerical viscosities. As the results were obtained for a two-dimensional Euler flow, the differences in these quantities depend solely on the numerical dissipation of the method and not on molecular dissipation and diffusion, or on physics due to vortex stretching present in three-dimensions. Most studies involving the solution of the Euler equations have focused on the numerical 'convergence' of some small set of quantities, such as the perturbation amplitude or mixing layer width in the Rayleigh-Taylor instability [5-7] and Richtmyer-Meshkov instability [8-10], over a limited range of evolution times with respect to grid refinement. The present work is conducted in the spirit of the investigation of double Mach reflection and Rayleigh-Taylor instability in two dimensions [11], which emphasized the computational advantage of higher order (ninth) WENO schemes over lower order (fifth) schemes for complex flows, mainly using qualitative comparisons. The present investigation is both quantitative and qualitative, and examines to what extent different orders of reconstruction and different resolutions capture physical quantities characterizing Richtmyer-Meshkov instability-induced mixing. The WENO method is well-suited for such an investigation, as it is possible to perform simulations identical in every other re- 
spect except for the order of reconstruction: this allows a self-consistent study distinct from utilizing entirely different numerical methods with different formal orders of spatial and temporal accuracy (see [12] where the results from a second-order centered essentially non-oscillatory method, a second-order Godunov method, and a spectral/compact finite-difference method applied to the two-dimensional single-mode Richtmyer-Meshkov instability without reshock are compared).

This paper is organized as follows. First, the numerical method and the set of simulations of the two-dimensional reshocked Richtmyer-Meshkov instability using different orders of WENO reconstruction and resolutions are summarized in Sec. 2, including a discussion of the benefits of formally high-order (highresolution) methods for investigating complex flows with shocks. The effects of order and resolution on the density, vorticity, simulated density Schlieren and baroclinic vorticity production fields are discussed in Sec. 3. The effects of order and resolution on the mixing layer width and circulation deposition are discussed in Sec. 4. The effects of order and resolution on the mixing profiles, mixing fractions, energy spectra, statistics, and probability distribution functions are presented in Sec. 5. As the results depend on the intrinsic numerical dissipation of the method, a quantitative estimate of the numerical dissipation obtained by computing the rate-of-change of the turbulent kinetic energy and numerical Reynolds numbers is presented in Sec. 6. The principal findings associated with each of the above are summarized at the end of each section or subsection. Finally, general conclusions, including a discussion of the relative computational cost of the simulations are presented in Sec. 7.

\section{The WENO method and two-dimensional simulations of reshocked single-mode Richtmyer-Meshkov instability}

The WENO reconstruction in the conservative finite-difference shock-capturing method used in the present study is briefly described, and its benefits for investigating complex hydrodynamic flows with shocks are discussed here. In particular, as shown later, formally higher-order reconstructions are less dissipative and have greater resolving power than lower-order ones. Thus, it is demonstrated that high-order WENO methods are suitable for investigating multi-dimensional shock-driven flows in which the dynamics of a wide range of scales and complex wave structures must be characterized with high fidelity. 


\subsection{Equations solved and description of the WENO algorithm}

In the present simulations, the Euler equations augmented by the mass fraction conservation equation for the second gas (used here to determine the mixing layer width and to quantitatively assess various mixing properties)

$$
\frac{\partial \boldsymbol{\phi}}{\partial t}+\frac{\partial \boldsymbol{F}}{\partial x}+\frac{\partial \boldsymbol{G}}{\partial y}=0
$$

are solved, where the conservative variables, $\boldsymbol{\phi}$, and inviscid fluxes, $\boldsymbol{F}$ and $\boldsymbol{G}$, are

$$
\begin{aligned}
& \boldsymbol{\phi}=(\rho, \rho u, \rho v, \rho e, \rho m)^{T}, \\
& \boldsymbol{F}=\left(\rho u, \rho u^{2}+p, \rho u v,(\rho e+p) u, \rho m u\right)^{T}, \\
& \boldsymbol{G}=\left(\rho v, \rho u v, \rho v^{2}+p,(\rho e+p) v, \rho m v\right)^{T} .
\end{aligned}
$$

Here, $\rho$ is the density, $\mathbf{u}=(u, v)$ is the velocity, $p$ is the pressure, $e=$ $\left(u^{2}+v^{2}\right) / 2+U$ is the total (kinetic plus internal) energy per unit mass, and $m$ is the mass fraction (here of the denser sulfur hexafluoride gas, $\mathrm{SF}_{6}$ ). The ideal gamma law gas pressure $p=\rho R T$ is used ( $R$ is the gas constant).

The numerical simulations of the reshocked Richtmyer-Meshkov instability were performed using the characteristic projection-based finite-difference WENO shock-capturing method [1]. In the WENO method, a piecewise-polynomial of degree $r$ is used to reconstruct the cell-average of the spatially-discretized characteristic variables of the Euler equations. As the polynomials may use stencils containing discontinuities and, thus, induce Gibbs oscillations in the solution, a weighted average of all of the possible polynomial reconstructions at a point is computed. Essentially zero weights are assigned to polynomials crossing discontinuities and nearly-equal weights are assigned to polynomials over smooth regions. With this weighting, the formal order of accuracy for the derivative of the conservative flux is $2 r-1$ in sufficiently smooth flow regions. In the present study, the semi-discrete equations are evolved in time using the third-order total variation diminishing (TVD) Runge-Kutta method [1].

The conservative finite-difference discretization of the Euler equations with WENO flux reconstruction contains implicit truncation errors that can be regarded as a nonlinear, adaptive numerical dissipation. Hence, the present simulations can be interpreted as a class of implicit large-eddy simulations (ILES) [13-16], in which the discrete equations are regarded as implicitly filtered and the implicit numerical dissipation is a surrogate for a dissipation provided by an explicit subgrid-scale model. As the non-dissipative compressible fluid dynamics equations are formally ill-posed, this numerical dissipation 
regularizes the method and renders it numerically stable for increasingly finer grids. As a result, quantities obtained from such simulations depend on the resolution and cannot be regarded as fully resolved.

\subsection{Benefits of the WENO method for simulating complex shocked flows}

Formally high-order methods better resolve complex flow features at long evolution times than traditional second- and third-order methods. It is also appreciated that higher order schemes are more computationally efficient than lower order schemes for the same accuracy $[17,18]$. In particular, sufficiently high order WENO methods are well-suited for the simulation of complex, compressible evolving flows containing shocks, other waves, and structures having a wide range of scales. The advantage of WENO methods is realized in complex multi-dimensional flows, as demonstrated in this paper for the reshocked Richtmyer-Meshkov instability.

The upwinding in the WENO method leads to large numerical dissipation in relatively smooth flow regions away from shocks. This dissipation can be reduced by hybridizing the WENO method with a high-order central difference scheme [19], and the resolving power can be improved by optimizing the stencil with a compact central-difference scheme in smooth flow regions [20]. A hybrid fifth-order compact upwind-WENO scheme was developed for shockturbulence interaction [21]. A hybrid scheme based on the weighted average of a compact scheme [21] and the fifth-order WENO scheme was subsequently developed [22] using a weight function that avoids the abrupt transition from one scheme to the other. A high-order accurate, hybrid central-WENO scheme was also recently developed [23].

Although two-dimensional Richtmyer-Meshkov flow was investigated in the present work, the code provides a robust MPI parallel framework for the multidimensional numerical simulation of the fully-nonlinear evolution of hydrodynamic instabilities and late-time mixing generated by single- or multi-mode Richtmyer-Meshkov, Rayleigh-Taylor and Kelvin-Helmholtz instabilities. The nonlinear system of hyperbolic partial differential equations can be solved in one, two or three spatial dimensions. The ratio of specific heats is assumed to be constant for both gases in the currently implemented (single-gamma) algorithm. As a result, some properties of the flow may not be predicted very accurately (i.e., transmitted and reflected shock speeds, time of reshock, initial interface velocity), but the mixing properties considered here are not expected to be strongly influenced by the single-gamma formulation, as the flow is nearly incompressible over most of the flow evolution for the Mach number considered [24]. 
Table 1

\begin{tabular}{|c|ccc|}
\hline \hline & Coarse (128) & Medium (256) & Fine (512) \\
\hline \hline Ninth-order & WENO9C & WENO9M & WENO9F \\
Fifth-order & WENO5C & WENO5M & WENO5F \\
Third-order & WENO3C & WENO3M & WENO3F \\
\hline
\end{tabular}

Keys used to denote simulations with different order of WENO flux reconstruction and grid resolution. The number in parenthesis refers to the number of grid points per initial perturbation wavelength $\lambda$.

\subsection{Simulations of reshocked single-mode Richtmyer-Meshkov instability}

Two-dimensional simulations of the reshocked Richtmyer-Meshkov instability modeled after the Mach 1.21 experiment of Collins and Jacobs [2,3] are presented and analyzed in the present work. The simulations are summarized in Table 1, where WENONX indicates the WENO method of order $\mathrm{N}=3$, 5 or 9 with optional resolution $\mathrm{X}=\mathrm{C}, \mathrm{M}$ or $\mathrm{F}$ (corresponding to coarse, medium and fine grid resolution, respectively), e.g., WENO5M is the fifthorder WENO method with 256 points per initial perturbation wavelength. Furthermore, order and resolution in the text always refer to order of WENO flux reconstruction and to the spatial grid resolution, respectively. In all cases, the simulations were performed to a late time of $18 \mathrm{~ms}$ to determine the effects of the interaction of the reflected rarefaction wave with the layer, and to investigate the late-time decay properties of the flow. The results presented in a previous study $[2,4]$ were obtained using WENO5M and WENO9M.

The initial conditions for the numerical simulations [2] were adapted from the Mach 1.21 air(acetone)/ $\mathrm{SF}_{6}$ experimental shock tube configuration of Collins and Jacobs [3]. As the current simulations use a single-gamma formulation, the experimental upstream conditions were matched. The adiabatic exponent $\gamma=1.24815$ was chosen, corresponding to the air(acetone) mixture. The preshock Atwood number was $A^{-}=\left(\rho_{\mathrm{SF}_{6}}^{-}-\rho_{\text {aa }}^{-}\right) /\left(\rho_{\mathrm{SF}_{6}}^{-}+\rho_{\text {aa }}^{-}\right)=0.604$. To match the dimensions of the shock tube test section, the computational domain had spanwise and streamwise length $L_{y}=8.9 \mathrm{~cm}$ and $L_{x}=78 \mathrm{~cm}$, respectively, with the perturbed initial interface located $3 \mathrm{~cm}$ from the edge of the domain (the physical spanwise domain was $17.8 \mathrm{~cm}$, and symmetry was used in the $y$ direction). An adaptive domain capability in the code allows the initial domain in $x$ to be much smaller than $L_{x}$. In the present simulations, the initial value $L_{x}=9.3 \mathrm{~cm}$ was chosen. The computational domain in the $x$ direction was elongated in $3 \mathrm{~cm}$ increments until a total length of $78 \mathrm{~cm}$ was attained. A CFL number of 0.45 was used in all of the simulations. The initial sinusoidal interface had amplitude $a_{0}=0.2 \mathrm{~cm}$ and wavelength $\lambda=5.9333 \mathrm{~cm}$. An initial diffusion layer thickness of $0.5 \mathrm{~cm}$ was used. 
The following boundary conditions were used: (1) periodic in the spanwise $(y)$ direction corresponding to the cross-section of the test section; (2) inflow at the test section entrance in the streamwise $(x)$ direction, and; (3) reflecting at the end wall of the test section in the streamwise direction. Additional experimental and numerical simulation details can be found elsewhere [2].

\section{The effects of order and resolution on the density, vorticity, simu- lated density Schlieren and baroclinic vorticity production fields}

As the Richtmyer-Meshkov instability develops, bubbles of air(acetone) 'rise' into the $\mathrm{SF}_{6}$ and spikes of $\mathrm{SF}_{6}$ 'fall' into the air(acetone). Following this initial growth, the spikes form roll-ups that develop the characteristic mushroom shape of the instability. Figure 1 shows the density and vorticity fields $\rho(x, y)$ and $\omega(x, y)$ at $6 \mathrm{~ms}$, before the shock reflected from the end wall reshocks the interface. The images were obtained by rotating the actual density and vorticity fields from the simulations $90^{\circ}$ counterclockwise. The density shows the spike of $\mathrm{SF}_{6}$ (red) with the characteristic roll-up, and the bubble of air(acetone) (blue) 'rising'. The vorticity shows the strong positive core rotating counter-clockwise (red) and the strong negative core rotating clockwise (blue), corresponding to the centers of the roll-ups. In general, as the order is increased and the grid is refined, the roll-up becomes better defined and sharper, and smaller-scale features appear within the roll-up. The vorticity shows similar features with more concentrated (smaller) cores. In the case of the WENO5M, WENO5F and WENO9 simulations, the roll-up contains a vortex bilayer with strong negative vorticity surrounded by a small layer of positive vorticity and vice versa. This vortex bilayer becomes sharper as the order and resolution are increased, and complex structures begin to form in the WENO9F simulation, manifested in the density as additional structures within the roll-up. Such structures have also been obtained in piecewiseparabolic method simulations [25,26], and is apparently a manifestation of a physical process observed in experiments [3,27-29]. A qualitative correspondence in both the density and vorticity occurs between simulations along a diagonal, so that doubling the resolution approximately corresponds to doubling the order (as also found in simulations of Rayleigh-Taylor instability and double Mach reflection [11]).

Figure 2 continues the comparison of the density and vorticity at $7 \mathrm{~ms}$, following reshock (which occurs at $\approx 6.6 \mathrm{~ms}$ ), with the bubble transforming into a spike and vice versa through an inversion process. The $\mathrm{SF}_{6}$ spike penetrates into the air(acetone) and becomes narrower as it transforms into a bubble. The inversion process is due to the deposition of vorticity of opposite sign during reshock. As the order is increased and the grid is refined, additional finerscale complex structures appear. This is also reflected in the vorticity, which 

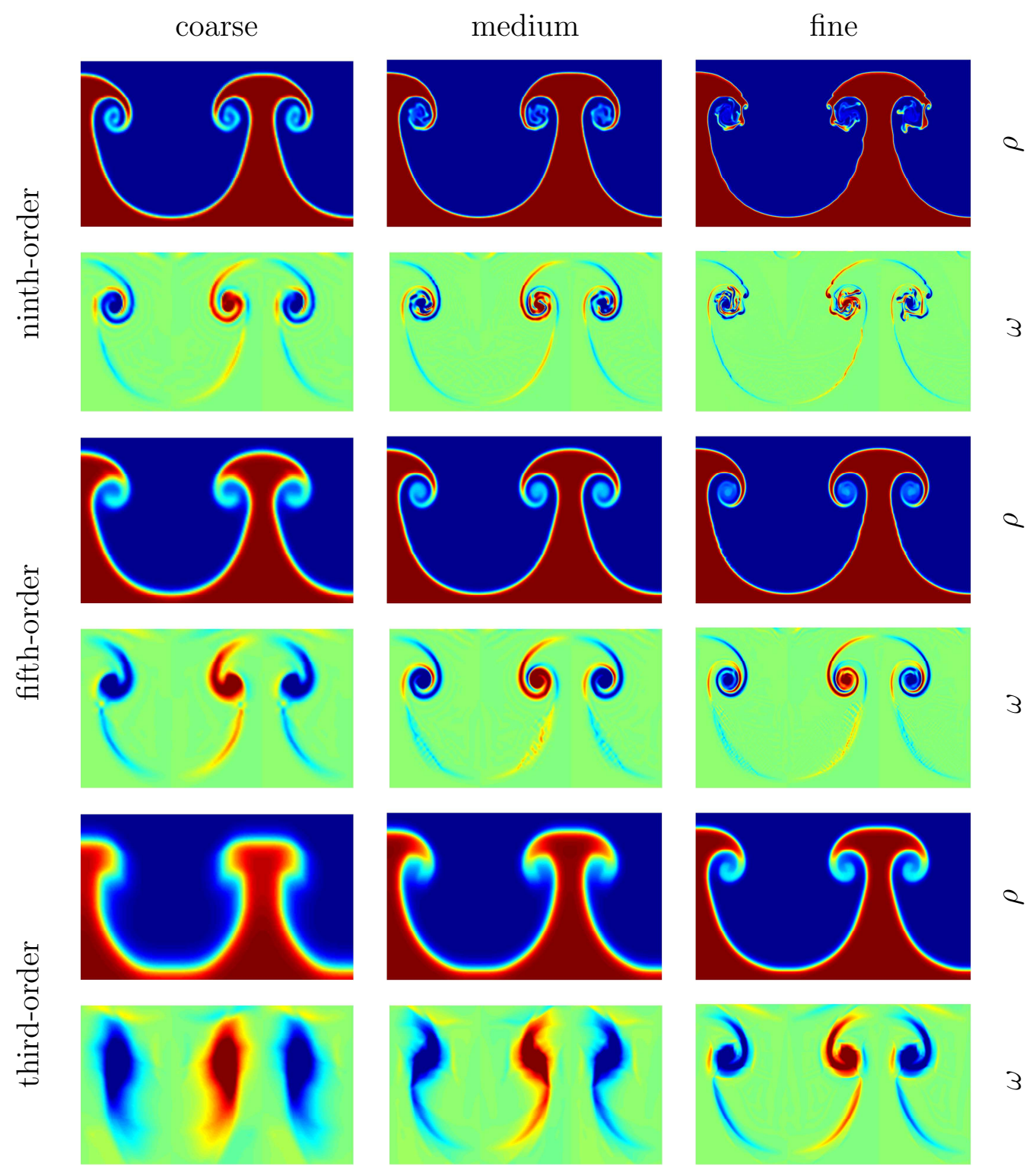

Fig. 1. The density and vorticity fields at $6 \mathrm{~ms}$ (before reshock) from the third-, fifth- and ninth-order simulations on the coarse, medium and fine grids.

shows that the flow transitions from strong positive and negative cores into vortex bilayers, and then into several fragmented cores. The figure presents an example of a complex flow with coherent large-scale structures.

Following reshock, a transmitted shock enters into the air(acetone) and a reflected rarefaction enters into the $\mathrm{SF}_{6}$ gas. As a result, a complex system of reflected and transmitted waves is observed at reshock that further contributes to the development of the instability. To visualize this system of waves, as well as the complex structures observed on the interface, the simulated density 

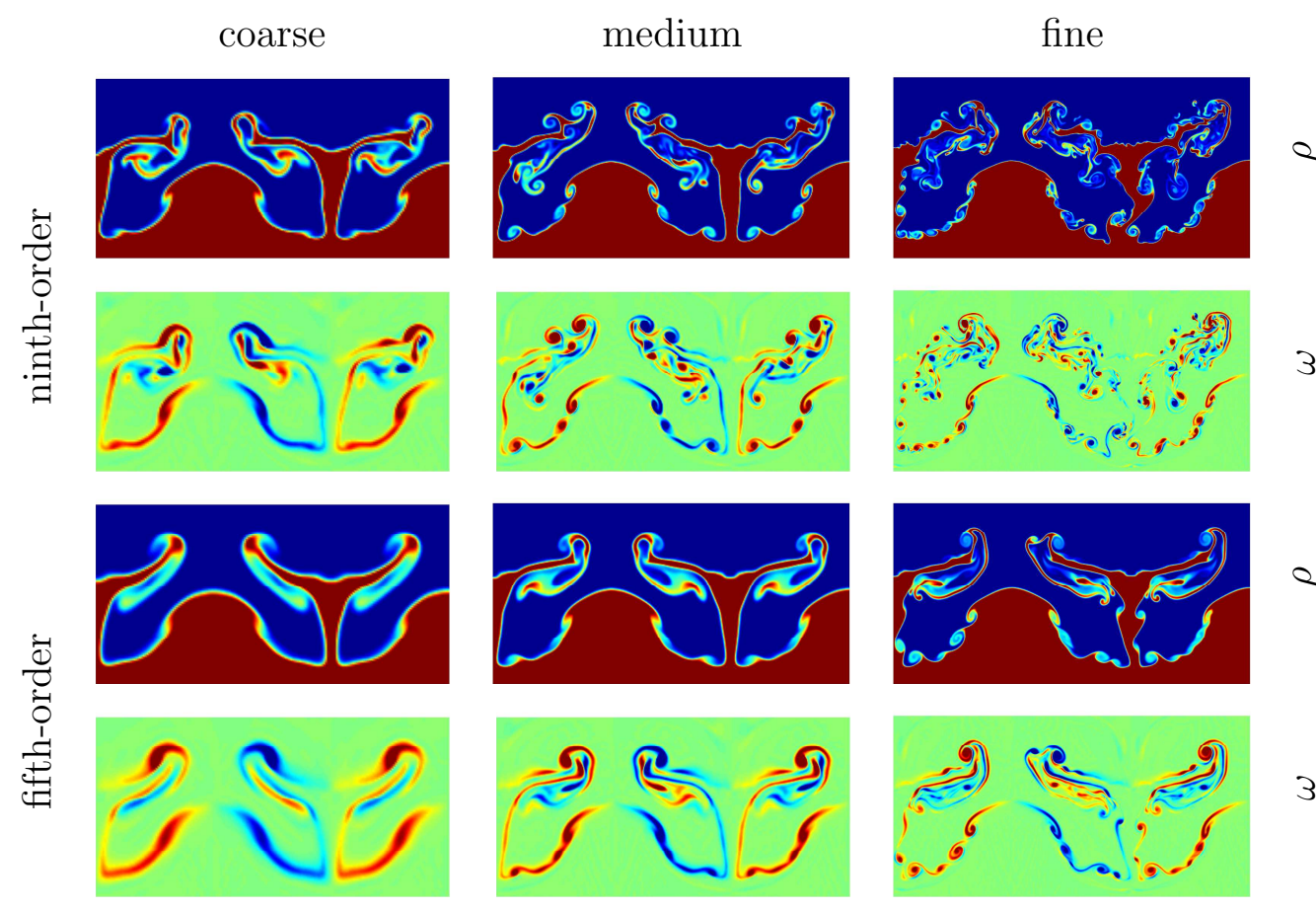

$$
\text { e }
$$
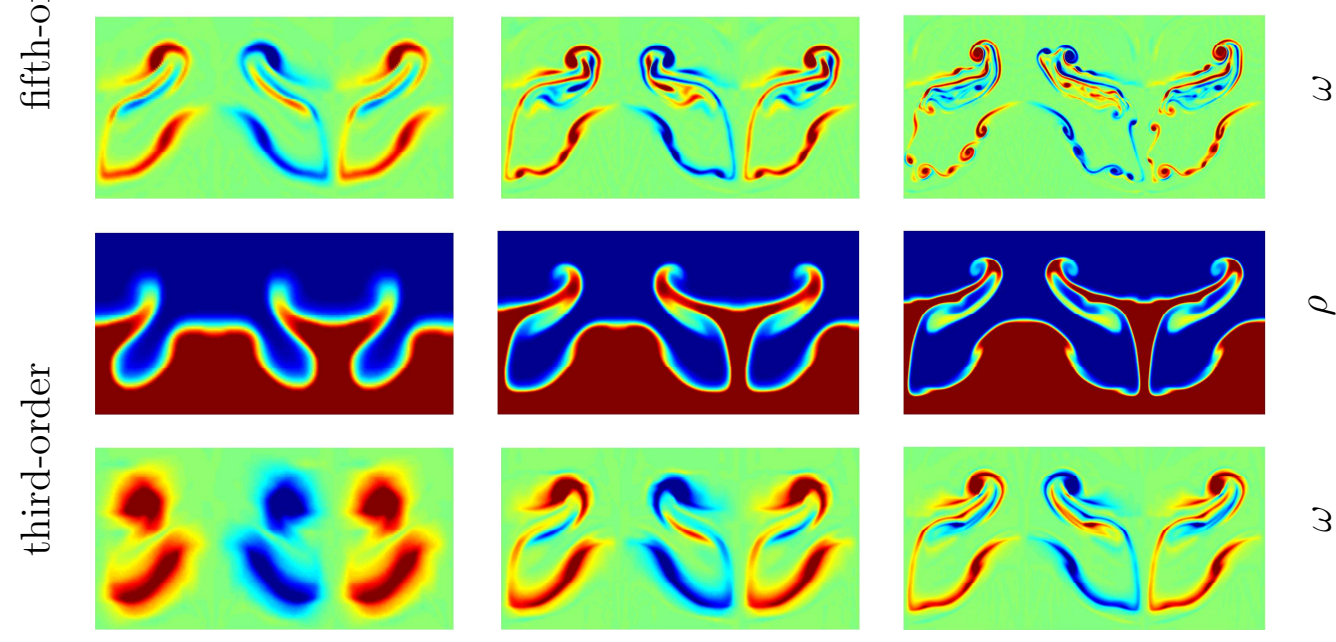

e 3

Fig. 2. The density and vorticity fields at $7 \mathrm{~ms}$ (after reshock) from the third-, fifthand ninth-order simulations on the coarse, medium and fine grids.

Schlieren fields [30]

$$
\Phi(x, y, t)=\exp \left[-\alpha(m) \frac{|\nabla \rho|}{\max |\nabla \rho|}\right], \alpha(m)= \begin{cases}20 & \text { if } m>m^{*} \\ 100 & \text { if } m<m^{*}\end{cases}
$$

where $m$ is the mass fraction of $\mathrm{SF}_{6}$ and $m^{*}=0.25$ is the threshold, are presented in Fig. 3 at $7 \mathrm{~ms}$. A complex system of curved intersecting waves is observed in the $\mathrm{SF}_{6}$ gas, corresponding to the reflected rarefaction, together with fine-scale structure surrounding the spike. As the order and resolution are increased, the waves become sharper and additional structures appear on the interface.

The density at $12 \mathrm{~ms}$ (late time following reshock) in Fig. 4 shows the evolution of the main spike, which develops several roll-ups and additional complex structure. The difference among the orders and resolutions is now significant. 
coarse
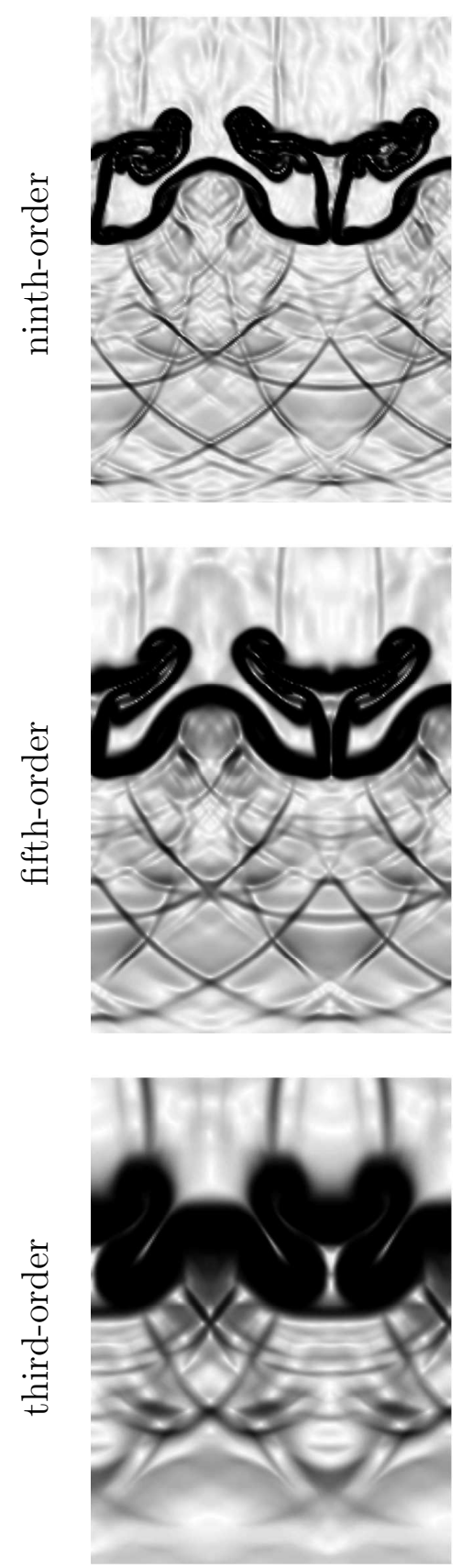

medium
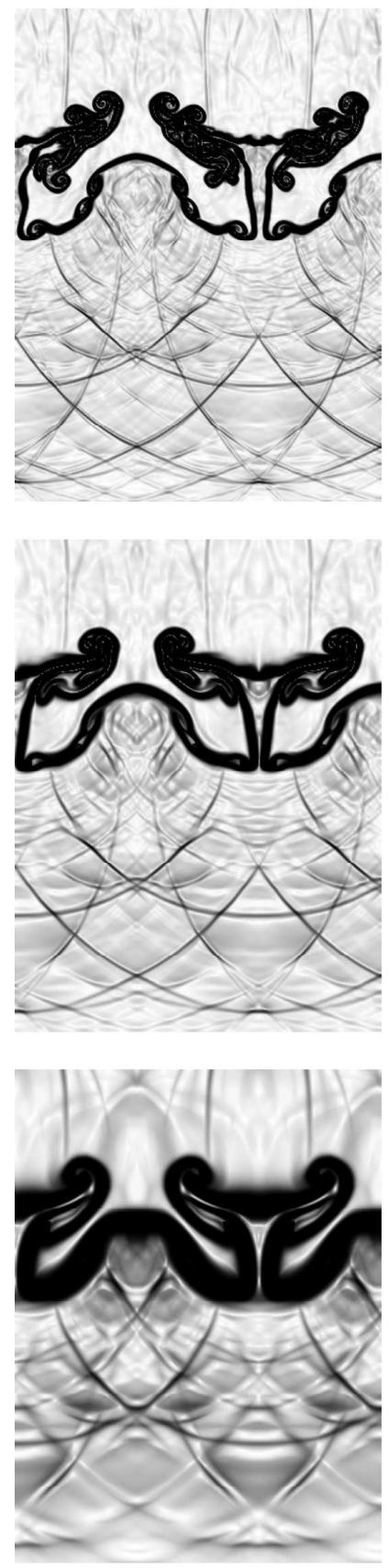

fine
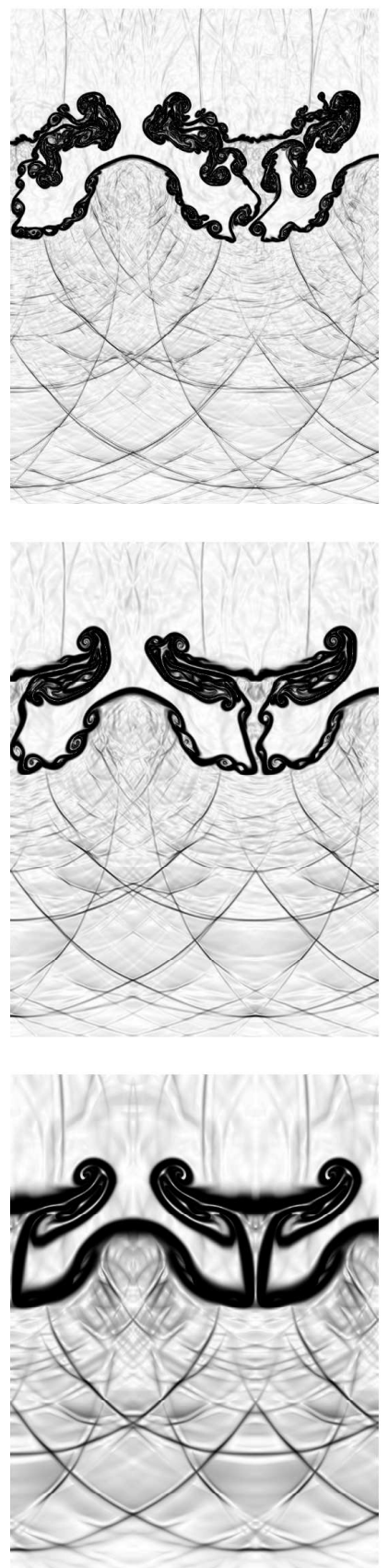

Fig. 3. The simulated density Schlieren fields at $7 \mathrm{~ms}$ (after reshock) from the third-, fifth- and ninth-order simulations on the coarse, medium and fine grids.

In particular, the WENO3 and WENO5C simulations retain significant coherency and symmetry, with a clearly identifiable dominant structure. Beginning with the WENO5M simulation, a progressive loss of symmetry occurs, together with the development of increasingly finer-scale structure. This is also reflected in the vorticity, in which large-scale organized strong cores are replaced by progressively fragmented and more concentrated structures. The 


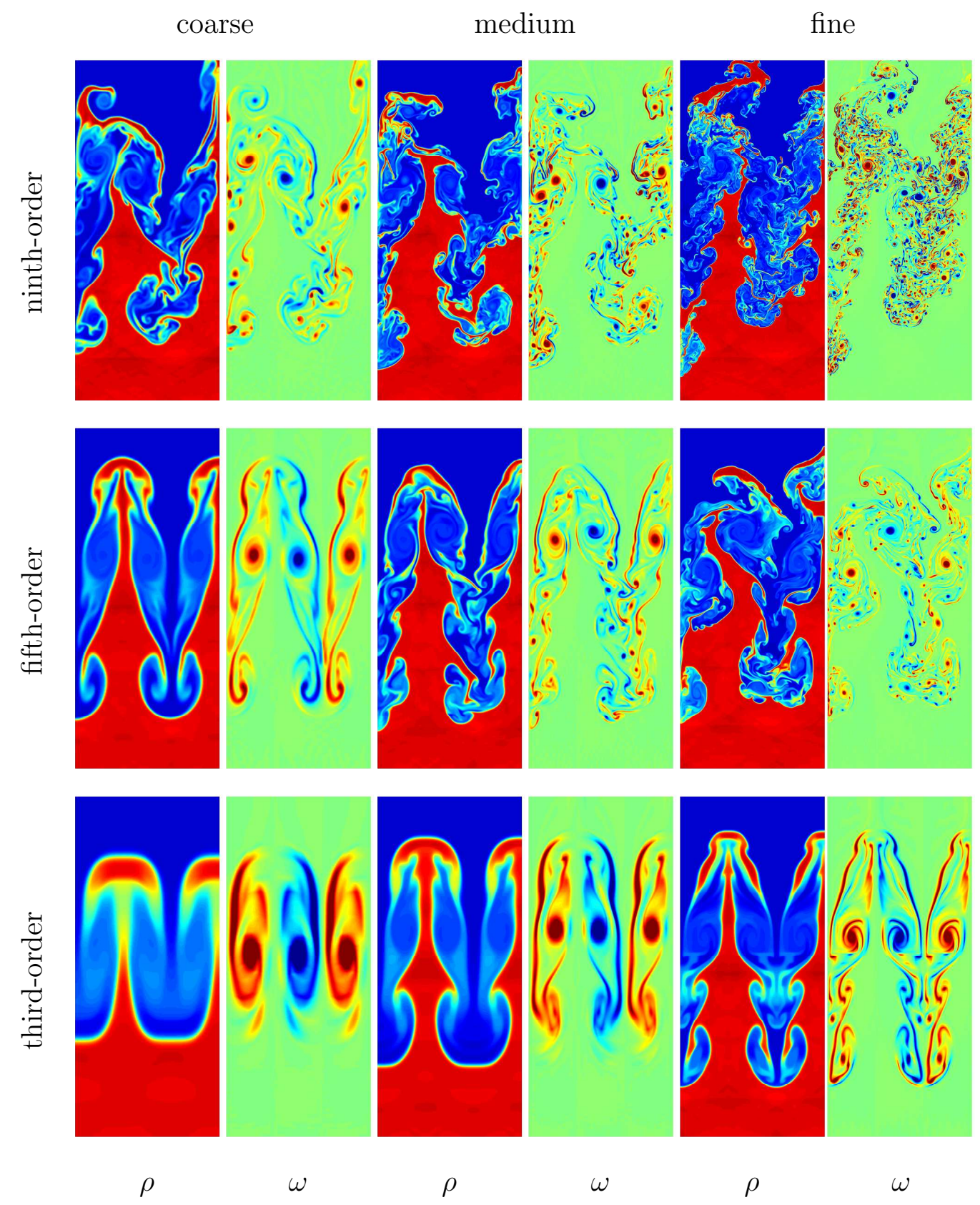

Fig. 4. The density and vorticity fields at $12 \mathrm{~ms}$ from the third-, fifth- and ninth-order simulations on the coarse, medium and fine grids.

dynamics of the vorticity is in qualitative agreement with two-dimensional turbulence phenomenology [4]. As the order and resolution are increased, the magnitude of the inverse kinetic energy cascade also decreases, resulting in the formation of more fragmented and smaller-scale structures. By contrast, as the resolution is decreased, the inverse kinetic energy cascade becomes stronger, leading to the formation of large persistent vortical structures. The WENO3 results are characteristic of simulations with a high degree of numerical diffusion, as well as of simulations that are spatially underresolved. Instead, the 

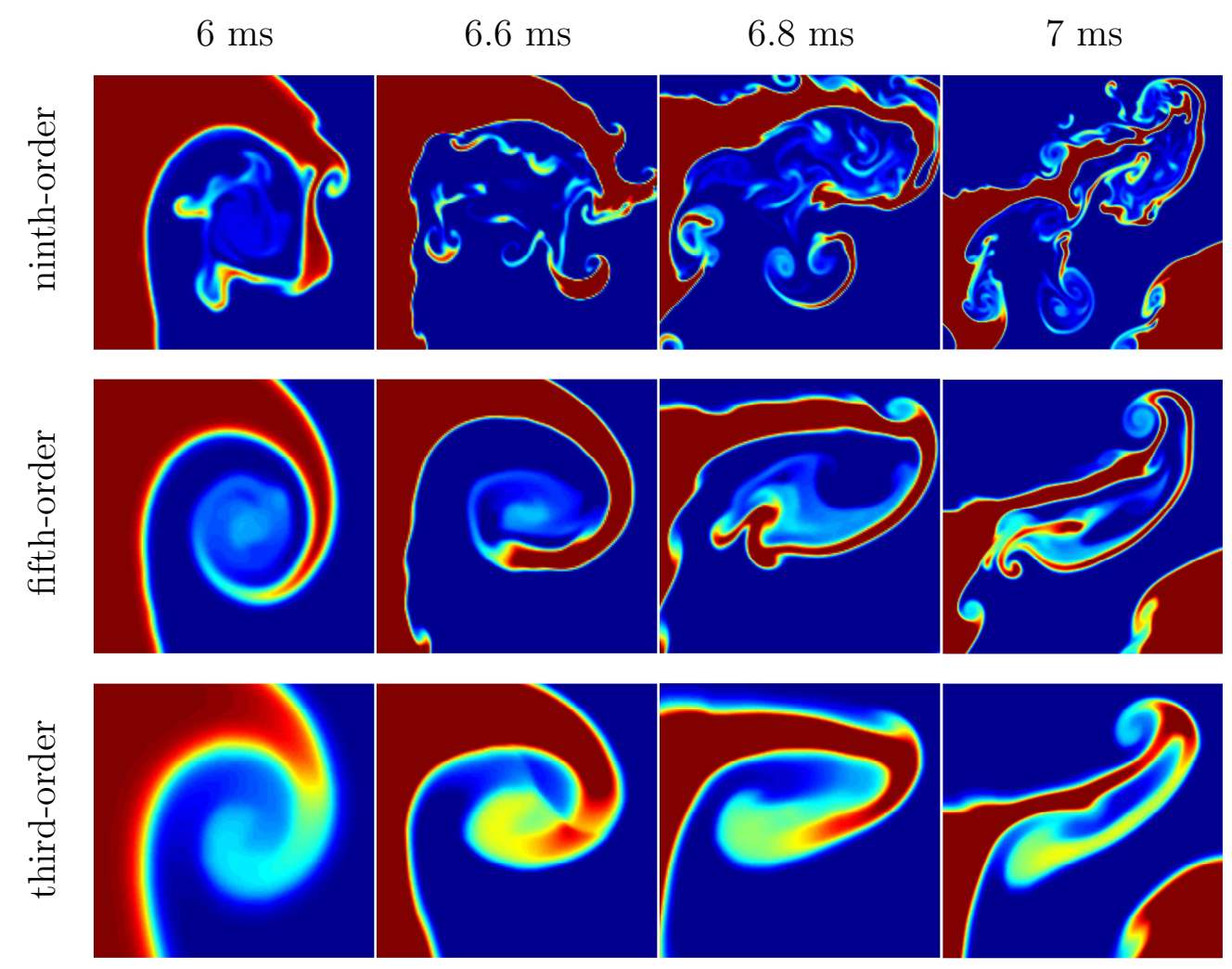

Fig. 5. Details of the roll-up in the density field from the ninth-, fifth- and third-order simulations on the fine grid at $6,6.6,6.8$ and $7 \mathrm{~ms}$.

fifth- and ninth-order simulations exhibit breaking of symmetry (reported in other simulations with reshock using lower order methods [10,31]), which can be attributed to numerical instabilities that are not damped by the intrinsic numerical dissipation in the WENO method.

Figure 5 illustrates the reshock process, showing the development of complex structures during the spike roll-up at 6, 6.6, 6.8 and $7 \mathrm{~ms}$ from the highresolution simulations. Prior to reshock at $6 \mathrm{~ms}$, the WENO9 simulations exhibit complex structures within the roll-up: such structures are visible in the inner core of the WENO5 simulations and are not present in the WENO3 simulations. During reshock, the roll-up is first compressed at $6.6 \mathrm{~ms}$ and then forms complex structures at later times. The WENO3 and WENO5 simulations retain a single dominant structure, while the WENO9 simulations exhibit fragmentation.

The spike dynamics during reshock can be further understood by considering the vorticity field $\omega(x, y)$ and the baroclinic vorticity deposition (production) $\mathcal{P}(x, y)$ during reshock. These quantities are shown in Fig. 6 at the same time as the density fields in Fig. 5. At $6 \mathrm{~ms}$, the vorticity from the WENO5F and WENO9F simulations show complex roll-up structures with regions of negative vorticity surrounded by positive vorticity forming a vortex bilayer. This 

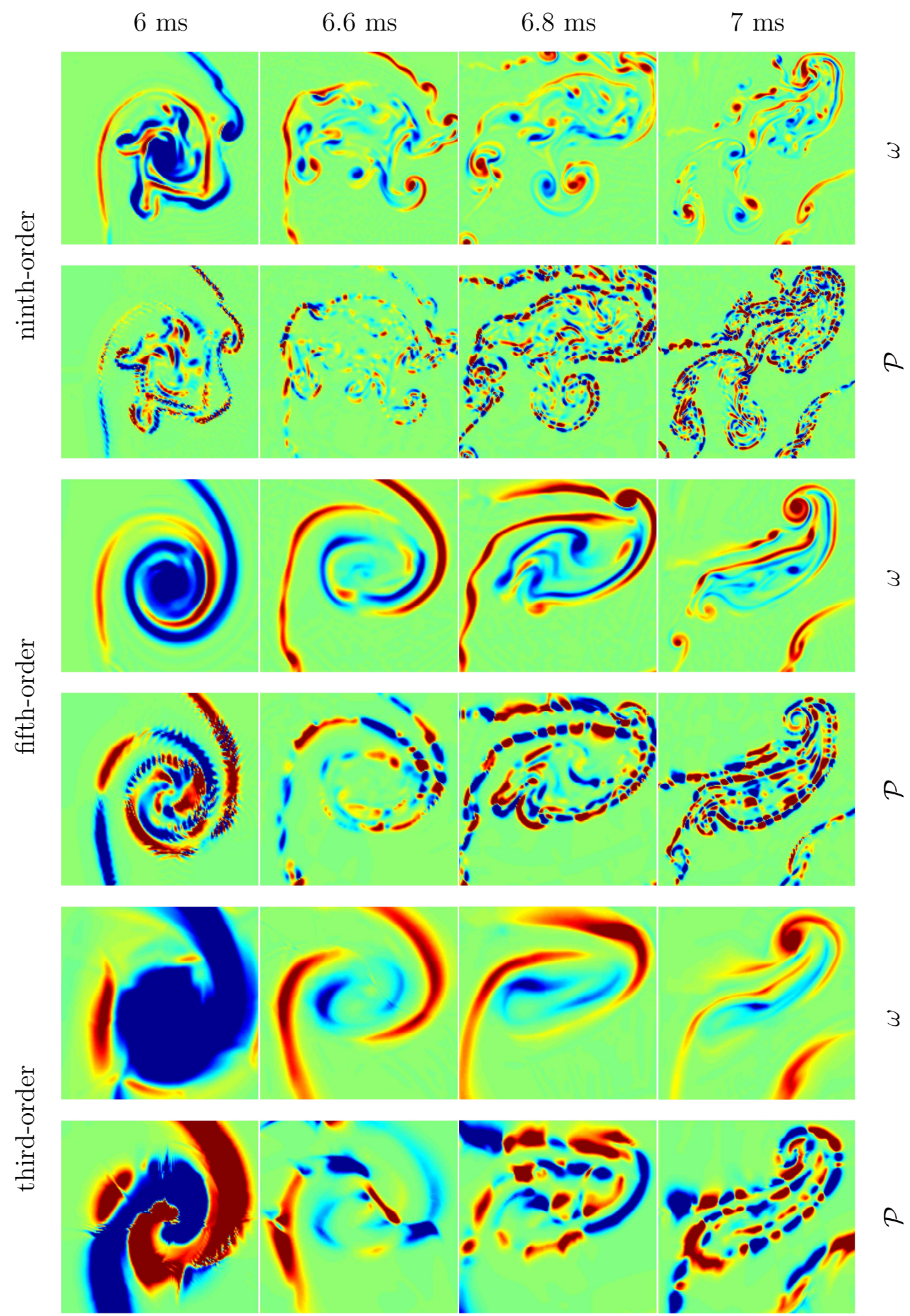

Fig. 6. Details of the roll-up in the vorticity and in the baroclinic vorticity production fields, $\omega$ and $\mathcal{P}$, from the ninth-, fifth- and third-order fine grid simulations at 6 , 6.6, 6.8 and $7 \mathrm{~ms}$. 
generation of positive vorticity is due to the baroclinic vorticity production mechanism. By contrast, the WENO3 simulations only show a strong negative core, and the baroclinic production does not exhibit the complex features observed in the WENO5 and WENO9 simulations. During reshock at $6.6 \mathrm{~ms}$, the strong negative core is significantly weakened and a layer of positive vorticity is deposited on the tip of the spike: this layer is particularly evident in the WENO3 and WENO5 simulations, and is thinner in the WENO9 simulations. The vorticity forms cores at 6.8 and $7 \mathrm{~ms}$ : the cores from the WENO9 simulations are more compact than those from the other simulations. This is also reflected in the baroclinic vorticity production, which is fragmented in the WENO9 simulations. The fields shown in Fig. 6 illustrate the significant difference in the vorticity dynamics for simulations including reshock.

In summary, prior to reshock, the density fields from higher-order higherresolution simulations exhibit additional finer-scale structure in the roll-up. The formation of this additional structure can be understood by considering the vorticity and the baroclinic vorticity production fields. The vorticity fields from higher-order higher-resolution simulations exhibit the formation of strong concentrated cores surrounded by a vortex bilayer of opposite sign, which is generated by the secondary baroclinic vorticity production activated by the misalignment of the density and pressure gradients as the roll-up occurs. This results in the fragmentation of the roll-up at late times, which is not observed in the lower-order lower-resolution simulations. The simulated density Schlieren fields were used to visualize the system of transmitted and reflected waves at reshock, including shock focusing observed in the reshocked spike. In higher-order higher-resolution simulations, the simulated density Schlierens exhibit sharper small-scale structure in the system of waves. Numerical dissipation damps the high frequency components of the wave structure, thus smearing the waves in the lower-order lower-resolution simulations. At late times, the density fields from higher-order higher-resolution simulations exhibit breaking of symmetry and the formation of complex fine-scale structures. Similarly, the vorticity fields exhibit fragmentation and the formation of localized cores. By contrast, the lower-order lower-resolution density fields exhibit symmetric large-scale structures with the vorticity fields having strong localized cores.

\section{The effects of order and resolution on the mixing layer width and circulation deposition}

The effects of order and resolution on the mixing layer width and the circulation deposition are considered in this section. It is shown, in general, that the dependence on order and resolution is most pronounced following reshock, with the differences among the simulations increasing with time. 


\subsection{The mixing layer width}

To determine the mixing layer width, first consider the mole fraction

$$
X(x, y, t)=\frac{m(x, y, t) M_{1}}{[1-m(x, y, t)] M_{2}+m(x, y, t) M_{1}},
$$

where $m$ is the mass fraction of $\mathrm{SF}_{6}$ and $M_{1}$ and $M_{2}$ are the molecular weights of air(acetone) and $\mathrm{SF}_{6}$, respectively. Spatially-averaging the mole fraction in the periodic $y$-direction gives

$$
\bar{X}(x, t)=\frac{1}{L_{y}} \int_{0}^{L_{y}} X(x, y, t) \mathrm{d} y,
$$

where $L_{y}$ is the spanwise width of the computational domain (shock tube). Finally, the spike and bubble locations, $\ell_{s}(t)$ and $\ell_{b}(t)$, are defined as the $x$ position where $\bar{X} \geq \epsilon$ and $\bar{X} \leq 1-\epsilon$, respectively, with $\epsilon=0.01$ in the present investigation (corresponding to a $1-99 \%$ criterion in the averaged mole fraction [32]). Therefore, the total mixing layer width is determined by the difference between the bubble and spike positions, $h(t)=\ell_{b}(t)-\ell_{s}(t)$.

Figure 7 shows the dependence of the mixing layer width $h(t)$ on the order and resolution up to $18 \mathrm{~ms}$ (left). Also shown are the widths normalized by the width from the WENO9F simulation (right). Prior to reshock, $h(t)$ is weaklydependent on both the order and resolution, with all simulations giving very similar widths: the flow is dominated by a single, large-scale evolving bubble (or spike), the front of which is not significantly affected by the dissipation. Following reshock, the dependence on order and resolution becomes significant, with $h(t)$ decreasing for lower orders and coarser resolutions. Prior to the arrival of the reflected rarefaction at $\approx 11 \mathrm{~ms}$, the mixing layer widths become closer as the order increases and the resolution is doubled. The differences are further amplified by the arrival of the reflected rarefaction and further increase later in time. At late times, the widths from the WENO3 simulations differ by as much as $\approx 5 \mathrm{~cm}$ as the grid is refined. By contrast, the widths obtained from the WENO5 and WENO9 simulations differ by $\approx 1 \mathrm{~cm}$. The normalized widths exhibit evidence for 'convergence' as the ratios approach unity for the WENO9 simulations.

In summary, the mixing layer width exhibits little dependence on the order and resolution prior to reshock. However, following reshock but prior to the arrival of the reflected rarefaction, large differences in the mixing layer widths are observed. As the order and resolution are increased, the differences between the widths decrease, suggesting a trend toward numerical 'convergence'. Following the arrival of the reflected rarefaction, the differences increase further. At late times, high-order high-resolution simulations display significant 


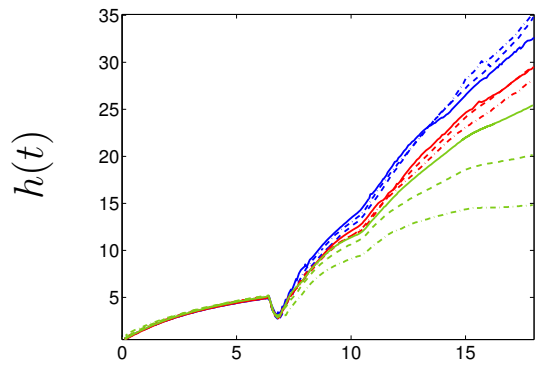

$t$

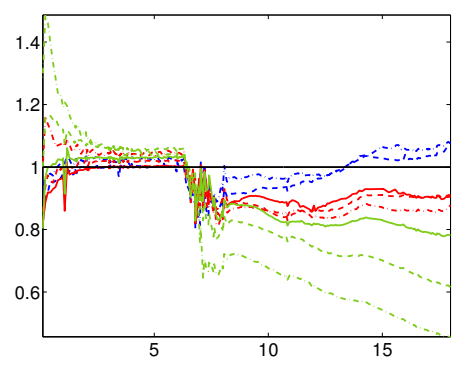

$t$

Fig. 7. Comparison of the time-evolution of the mixing layer width when the order and resolution are varied (left). The mixing layer widths normalized by that from the WENO9F simulation are also shown (right). Quantities obtained using the WENO3, WENO5 and WENO9 simulations are shown in green, red and blue, respectively; quantities obtained on the fine, medium and coarse grid are shown using a solid, dashed and dash-dot line, respectively.

fragmentation of the complex structures, causing small oscillations in $h(t)$ as the cutoff limit used in the determination of the width is approached. The differences in the widths following reshock can be attributed to the effects of the implicit numerical dissipation in the WENO method, which suppresses the formation of small-scale structures in low-order low-resolution simulations. Small-scale structures do not have a direct effect on the width prior to reshock. However, the energy deposited into the layer by the reshock process generates small-scale structures, which explains the differences in widths as less energy is deposited in low-order low-resolution simulations.

\subsection{The interfacial circulation deposition}

The vorticity deposited by the shock on the interface, as well as the additional deposition due to the baroclinic vorticity production, can be quantified using the positive and negative circulation. As the vorticity field and the outward area element are parallel, the positive and negative circulations $\Gamma^{ \pm}$can be approximated on the rectangular grid with spacing $\Delta x$ and $\Delta y$ over $U^{+}=$ $\left\{\lambda / 2<y_{j}<\lambda\right\}$ and $U^{-}=\left\{y_{j}<\lambda\right\}[4]$ :

$$
\Gamma^{ \pm}(t)=\sum_{i=1}^{N_{x}} \sum_{j \in U^{ \pm}, j=1}^{N_{y}} \omega\left(x_{i}, y_{j}, t\right) \Delta x \Delta y
$$

Before reshock, the positive circulations $\Gamma^{+}$in Fig. 8 are qualitatively similar: a steady increase due to baroclinic vorticity production is observed. As the spike rolls up and vorticity of opposite sign is created inside the roll-ups (forming vortex bilayers), the circulation stops increasing and a slight decrease is 


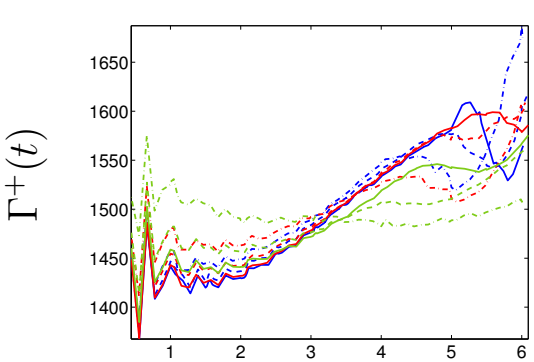

$t$

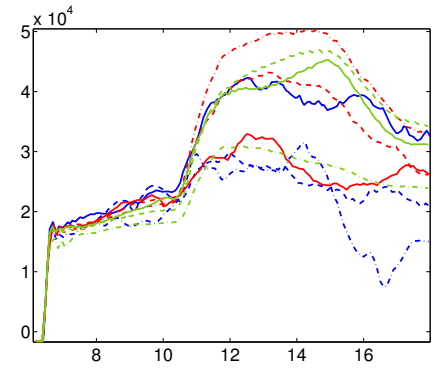

$t$

Fig. 8. Comparison of the time-evolution of the positive circulation $\Gamma^{+}$before reshock (left) and after reshock (right) when the order and resolution are varied. See Fig. 7 for the legend.

observed in the case of the WENO9F simulation. Instead, the WENO3C circulation is nearly constant across the layer, as the baroclinic vorticity production is small. A sharp increase in the circulation occurs at reshock, corresponding to the rapid deposition of additional vorticity on the interface. Following reshock and prior to the arrival of the reflected rarefaction at $\approx 11 \mathrm{~ms}$, the circulations from all of the simulations are qualitatively similar, exhibiting a steady increase due to the additional deposition of vorticity. After the arrival of the reflected rarefaction, symmetry breaks and the regions $U^{+}$and $U^{-}$no longer contain vorticity of a single sign, resulting in large differences in the circulations for times greater than $11 \mathrm{~ms}$.

\section{The effects of order and resolution on mixing profiles, chemical products, mixing fractions, energy spectra, statistics and prob- ability distribution functions}

The effects of the order and resolution on mixing profiles, chemical products, mixing fractions, energy spectra, statistics and probability distribution functions are quantified in this section. The comparisons of the effects of order and resolution are conducted at $6 \mathrm{~ms}$ immediately before reshock, at $7 \mathrm{~ms}$ immediately after reshock, and at $18 \mathrm{~ms}$ at late times following reshock. To facilitate the comparison of the quantities, the streamwise coordinate is recentered by the midpoint $x_{\text {mid }}(t)$ between the bubble and spike position, and normalized by the mixing layer width $h(t)$ : the mixing layer is mapped to $\left[x-x_{\text {mid }}(t)\right] / h(t) \in[-0.5,0.5]$. Most of the effects on the quantities considered can be interpreted in terms of the relative numerical dissipation, with increasing order and increasing resolution associated with decreasing numerical dissipation. The results presented here demonstrate that different quantitative characterizations of mixing are possible depending on the order and resolution. As a result, caution must be exercised when interpreting the results 
$6 \mathrm{~ms}$
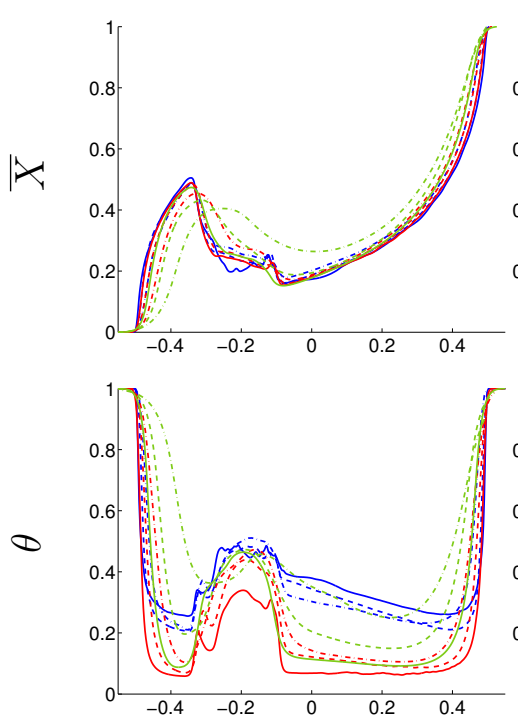

$$
\left[x-x_{m i d}(t)\right] / h(t)
$$

$7 \mathrm{~ms}$
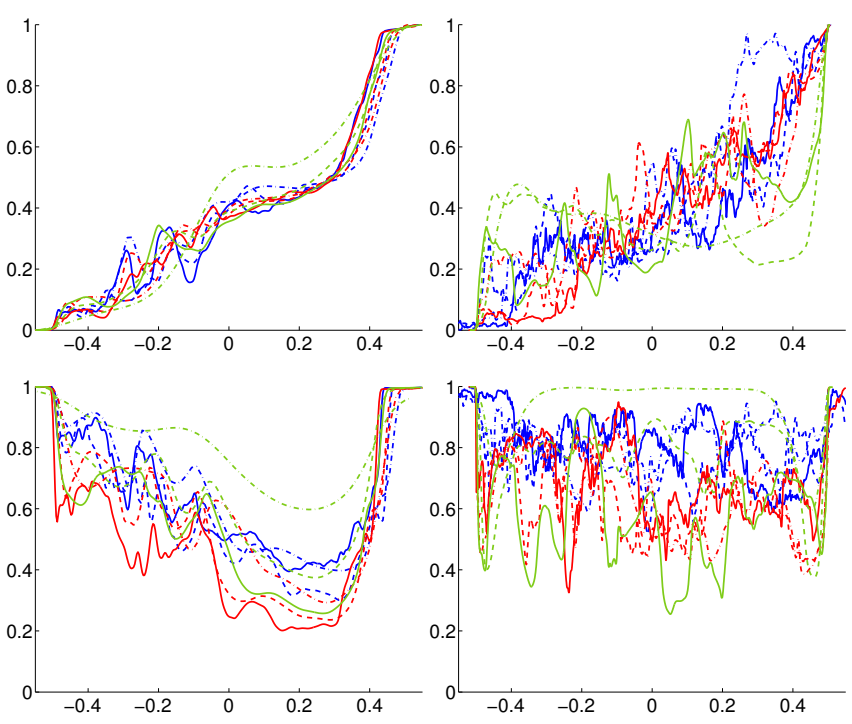

$\left[x-x_{m i d}(t)\right] / h(t)$

Fig. 9. Comparison of the averaged mole fraction profile $\bar{X}$ and molecular mixing profile $\theta$ across the layer at 6,7 and $18 \mathrm{~ms}$ when the order and resolution are varied. See Fig. 7 for the legend.

of numerical simulations of the Richtmyer-Meshkov instability as described by the Euler equations.

\subsection{The mixing profiles}

Up to $7 \mathrm{~ms}$, the mole fraction profiles $\bar{X}(x, t)$ in Fig. 9 agree at sufficiently high resolution. A more disordered structure is observed at late times. As $\bar{X}$ measures the distribution of mass across the mixing layer, the differences observed at $18 \mathrm{~ms}$ are due to different mass distributions at late times among the different simulations.

Figure 9 also shows the molecular mixing profile [33,34]

$$
\theta(x, t)=\frac{\overline{f_{1} f_{2}}}{\overline{f_{1}} \overline{f_{2}}} \quad \text { where } \quad f_{1}(x, y, t) \equiv \frac{\frac{m(x, y, t)}{\rho_{1}}}{\frac{m(x, y, t)}{\rho_{1}}+\frac{1-m(x, y, t)}{\rho_{2}}}
$$

and $f_{2}=1-f_{1}$ are the volume fractions. Here, $\bar{\phi}$ signifies the spatial average of any field $\phi(x, y, t)$ over the periodic $y$-direction. The molecular mixing profile $\theta(x, t)$ exhibits strong sensitivity to the order and resolution. A large spike is observed on the air(acetone) side at $6 \mathrm{~ms}$, corresponding to the roll-up of the spike: all of the simulations approximately capture the effect of this large spike. In the regions near the stem of the spike, the WENO9 simulations yield 
$6 \mathrm{~ms}$
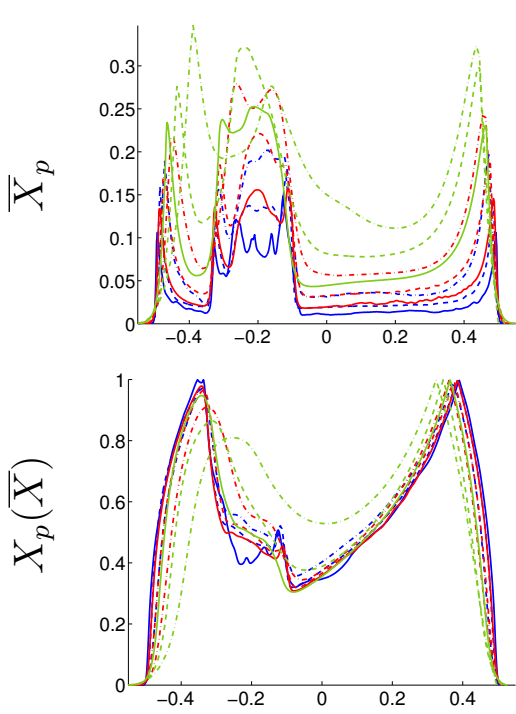

$$
\left[x-x_{m i d}(t)\right] / h(t)
$$

$7 \mathrm{~ms}$
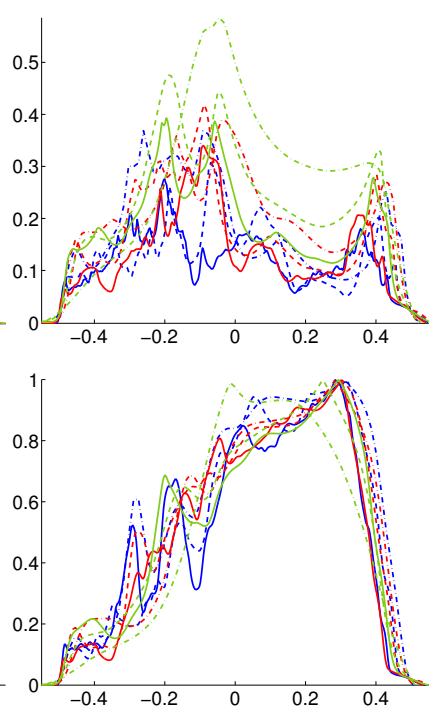

$\left[x-x_{m i d}(t)\right] / h(t)$
$18 \mathrm{~ms}$
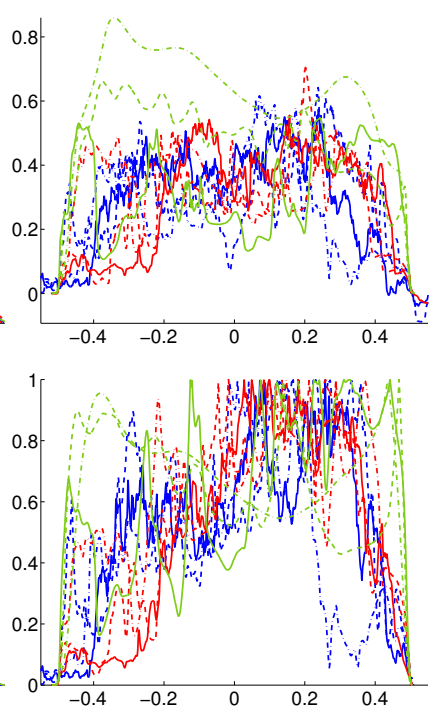

$\left[x-x_{m i d}(t)\right] / h(t)$

Fig. 10. Comparison of the averaged product mole fraction profile $\bar{X}_{p}$ and maximum product mole fraction profile $X_{p}(\bar{X})$ across the layer at 6,7 and $18 \mathrm{~ms}$ when the order and resolution are varied. See Fig. 7 for the legend.

larger values and a sharper transition near the boundaries than the WENO3 and WENO5 simulations. This is consistent with the higher-order simulations having sharper interfaces with less numerical diffusion. The increased value of $\theta$ at $7 \mathrm{~ms}$ results from the compression caused by reshock. The profile is also shifted toward the air(acetone) side, corresponding to the increased mixing near the spike, while less mixing occurs as the new spike of $\mathrm{SF}_{6}$ (due to the inversion process) develops. The simulations qualitatively capture the same overall behavior, but the differing values indicate increased sensitivity. A similar remark also applies at $18 \mathrm{~ms}$, which also shows the effects of the profoundly different distribution of mass and mixing across the layer at late times among the simulations.

A quantitative measure of mixing can be defined as follows. Suppose that the two fluids undergo a fast kinetic reaction, so that the amount of product produced is $[32,35]$

$$
X_{p}(x, y, t)= \begin{cases}\frac{X(x, y, t)}{X_{s}} & \text { if } X \leq X_{s} \\ \frac{1-X(x, y, t)}{1-X_{s}} & \text { if } X>X_{s}\end{cases}
$$

with $X_{s}=1 / 2$, indicating that the product is composed of one mole of each 'reactant', and is limited by the amount of reactant (either the heavy or the light fluid). Figure 10 shows the averaged product mole fraction profile $\bar{X}_{p}(x, t)$, which provides a measure of how well mixed the two 'reactants' are. Values near unity indicate that complete mixing has occurred resulting in the forma- 
tion of chemical product, while values near zero correspond to little mixing and little chemical product formation. Here, numerically-induced mixing is a surrogate for chemical reactions. At $6 \mathrm{~ms}, \bar{X}_{p}$ exhibits a large peak corresponding to the roll-up of the spike. The magnitude of the peak shows that the WENO9, WENO5 and WENO3 simulations correspond to progressively increased mixing in the roll-up. Similar behavior is also observed along the stem of the spike and at the tip of the bubble and spike. These results are consistent with a reduced numerical diffusion in the WENO9 simulations, and therefore of diffusion-induced mixing in higher-order simulations. At $7 \mathrm{~ms}$, the profile shows a peak near the air(acetone) side corresponding to the compressed spike roll-up and a smaller value near the $\mathrm{SF}_{6}$ side, corresponding to the development of the spike due to the inversion process. At $18 \mathrm{~ms}$, the profile is nearly uniform across the layer but with additional features, associated with the different underlying mixing profiles at late times.

Also shown in Fig. 10 is the maximum product mole fraction profile $X_{p}(\bar{X})$, which physically corresponds to the total amount of product obtained if both fluids were homogeneously mixed. At $6 \mathrm{~ms}, X_{p}(\bar{X})$ exhibits large peaks near the bubble and spike tips, corresponding to the small diffusive mixing in these locations. A small peak corresponding to the center of the roll-up is captured by all of the simulations, with the exception of the WENO9F simulation, which results from the additional structure within the roll-ups. At $7 \mathrm{~ms}$, all of the profiles are very similar and have a large peak close to the $\mathrm{SF}_{6}$ side, corresponding to the development of the spike. The oscillatory profiles at 18 ms result from the underlying small-scale structure within the mixing layer.

In summary, the mixing profiles at $6 \mathrm{~ms}$ (prior to reshock), at $7 \mathrm{~ms}$ (immediately following reshock) and at $18 \mathrm{~ms}$ (late time) exhibit different degrees of sensitivity to the order and resolution. Prior to and immediately following reshock, the differences are due to the order and resolution, i.e., the magnitude of the numerical diffusion. However, the differences in the mixing fractions at late times are also due to significant differences in the underlying flow structure. In particular, higher-order higher-resolution simulations exhibit fragmented structured with localized mixed regions, resulting in profiles with more pronounced localized peaks. By contrast, lower-order lower-resolution simulations exhibit more coherent large-scale structures, resulting in fewer peaks spread over a larger portion of the mixing layer. As a result, the mole fraction profile $\bar{X}$ measuring the mass distribution shows similar values as the resolution and order are increased prior to and immediately following reshock. By contrast, the averaged product mole fraction profile $\bar{X}_{p}$, which quantifies the mixing if the two gases were 'reacting', exhibits differences for all times prior to and following reshock. 

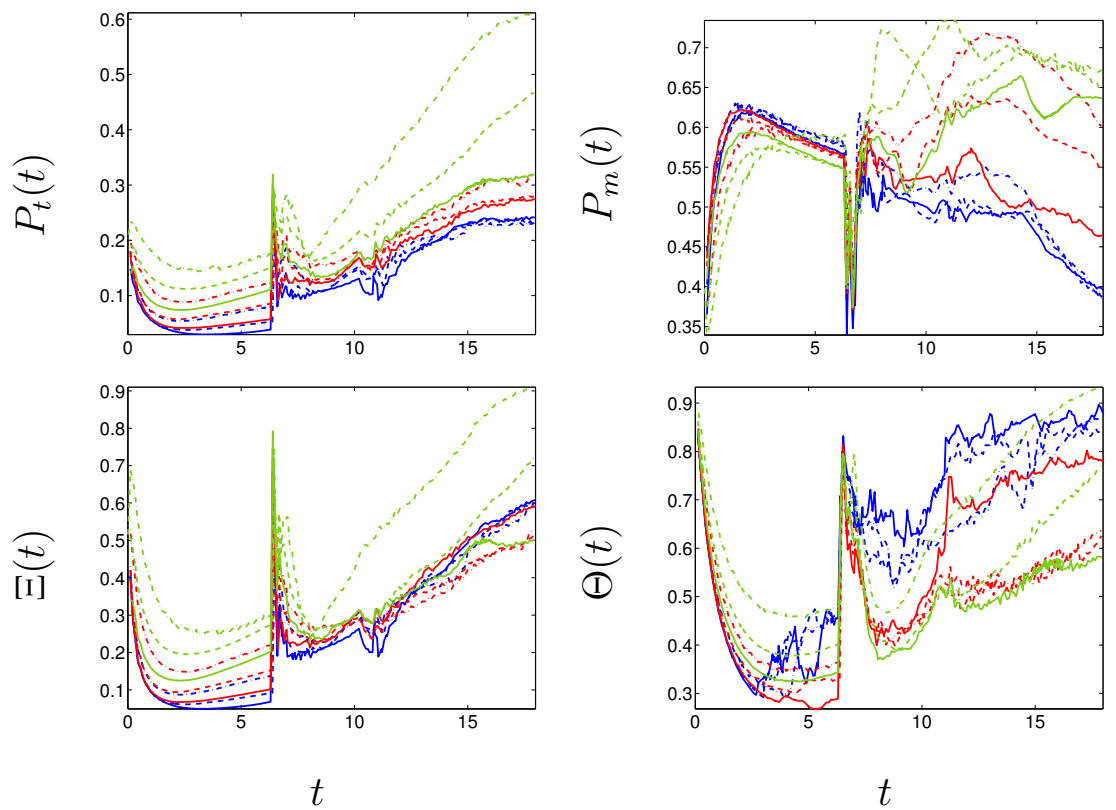

Fig. 11. Comparison of the time-evolution of the chemical products and mixing fractions $P_{t}(t), P_{m}(t), \Xi(t)$ and $\Theta(t)$ when the order and resolution are varied. See Fig. 7 for the legend.

\subsection{The chemical products and mixing fractions}

Using Eq. (11), the total chemical product $P_{t}(t)$, maximum chemical product $P_{m}(t)$, and mixing fractions $\Xi(t)$ and $\Theta(t)$ can be defined as

$$
\begin{aligned}
& P_{t}(t)=\int_{\ell_{s}(t)}^{\ell_{b}(t)} \overline{X_{p}} \mathrm{~d} x, \quad P_{m}(t)=\int_{\ell_{s}(t)}^{\ell_{b}(t)} X_{p}(\bar{X}) \mathrm{d} x, \\
& \Xi(t)=\frac{P_{t}(t)}{P_{m}(t)}, \quad \Theta(t)=\frac{\int_{\ell_{s}(t)}^{\ell_{b}(t)} \overline{f_{1} f_{2}} \mathrm{~d} x}{\int_{\ell_{s}(t)}^{\ell_{b}(t)} \overline{f_{1}} \overline{f_{2}} \mathrm{~d} x},
\end{aligned}
$$

respectively; $P_{m}(t)>P_{t}(t)$ is the amount of product obtained if both 'reactants' were homogeneously mixed. Values near unity correspond to complete mixing for both $\Xi$ and $\Theta$, while values near zero correspond to little mixing.

The total chemical product $P_{t}(t)$ in Fig. 11 exhibits a rapid decrease following the initial passage of the shock, followed by a steady increase. Reshock causes a sharp peak followed by a rapid decrease. A steady increase occurs following reshock, with a small decrease corresponding to the arrival of the reflected rarefaction wave at $\approx 11 \mathrm{~ms}$. The reflected rarefaction stretches the interface but does not contribute to mixing. Consequently, $\bar{X}_{p}$ is expected to decrease due to the arrival of the reflected rarefaction, resulting in smaller values of $P_{t}$. All of the simulations exhibit similar qualitative behavior, with the WENO3, WENO5 and WENO9 simulations giving progressively smaller values resulting 
from the correspondingly smaller values of $\bar{X}_{p}$.

Figure 11 also shows that the maximum chemical product $P_{m}(t)$ exhibits different behavior than $P_{t}(t)$. The passage of the incident shock causes a rapid increase followed by a steady decrease: the decrease is caused by the formation of the spike which effectively "demixes" the initially mixed interface. Reshock causes a rapid decrease, followed by a rapid increase. Following reshock, the values are quite different. The WENO3 simulations show a rapid increase followed by a steady decrease. The WENO5C and WENO5M simulations show an increase until $\approx 11 \mathrm{~ms}$, followed by a decrease; the WENO5F and WENO9 simulations show a steady decrease. This behavior is a consequence of the different structures in the mixing layer following the passage of the shock. In particular, the WENO9 simulations show features in largely unmixed regions. By contrast, the WENO3 simulations show significant mixing at all times.

The mixing fractions $\Xi(t)$ and $\Theta(t)$ are also shown in Fig. 11. Although both quantities provide similar insight into the mixedness of the fluids, the results obtained using different orders and resolutions exhibit significant differences. First, $\Xi$ rapidly decreases following passage of the incident shock, and then steadily increases. Reshock causes a rapid increase followed by a rapid decrease, and then a steady increase indicating increased mixing near the interface. The values of $\Xi$ can be interpreted in terms of the behavior of the total and maximum chemical product, $P_{t}$ and $P_{m}$. By contrast, $\Theta$ exhibits different behavior than $\Xi$. Following the passage of the shock, $\Theta$ rapidly decays and then increases. It is noteworthy that the WENO9F simulation exhibits quite different behavior as $\Theta$ is influenced by the structures within the roll-ups. Reshock rapidly increases $\Theta$, followed by a rapid decrease in the WENO3 and WENO5 simulations. The decrease is not as large in the WENO9 simulations. A steady increase in $\Theta$ then follows. These results are again a consequence of the different structures in the flow following reshock. In particular, the large unmixed regions in the WENO9 simulations contribute to larger values of $\Theta$.

In summary, the chemical products and mixing fractions exhibit a significant dependence on the order and resolution. Low-order low-resolution simulations have increased numerical dissipation, resulting in greater overall numerical mixing as measured by $P_{t}$ both before and after reshock. High-order highresolution simulations have smaller numerical dissipation, resulting in smaller values of $P_{t}$. Before reshock, the values of the maximum production fraction $P_{m}$ are similar for all simulations, consistent with similar widths of the mixing layer and similar structures as the instability develops. Following reshock, the mixing layer width from high-order high-resolution simulations is larger and causes the value of $P_{m}$ to decrease. High-order high-resolution simulations having the largest mixing layer widths exhibit the smallest values of $P_{m}$. This change in behavior following reshock is reflected in the mixing fraction $\Xi$. Prior to reshock, low-order low-resolution simulations have larger values of $\Xi$, con- 
sistent with increased overall mixing due to the larger numerical dissipation. However, following reshock, $\Xi$ is not very sensitive to the order and resolution. Similar mechanisms also explain the behavior of the molecular mixing fraction $\Theta$, which exhibits strong sensitivity to the order and resolution.

\subsection{The energy spectra}

The effects of order and resolution on the distribution of energy among different scales can be quantified by considering energy spectra associated with different fluctuations. Let $\bar{\phi}(x, t)$ denote an instantaneous average of a field $\phi(x, y, t)$ over the periodic (spanwise) direction $y$ with length $L_{y}$. Define the corresponding fluctuating field $\phi(x, y, t)^{\prime} \equiv \phi(x, y, t)-\bar{\phi}(x, t)$. Introduce the instantaneous density-weighted average $\widetilde{\phi}(x, t) \equiv \overline{\rho \phi} / \bar{\rho}$, and the corresponding fluctuating field $\phi(x, y, t)^{\prime \prime} \equiv \phi(x, y, t)-\widetilde{\phi}(x, t)$. The energy associated with each Fourier mode $k$ is obtained by averaging over the extent of the mixing layer to obtain the one-dimensional energy spectrum of $\phi$,

$$
E_{\phi \phi}(k, t)=\frac{1}{2 h(t)} \int_{\ell_{s}(t)}^{\ell_{b}(t)}|\widehat{\phi}(k, x, t)|^{2} \mathrm{~d} x
$$

where $\widehat{\phi}(k, x, t)=\frac{1}{2 \pi} \int_{-\infty}^{\infty} \phi(x, y, t) \exp (-i k y) \mathrm{d} y$ is the Fourier transform of $\phi(x, y, t)$ in the periodic spanwise direction.

The streamwise turbulent kinetic energy spectrum $E_{u^{\prime \prime} u^{\prime \prime}}(k, t)$ in Fig. 12 is weakly dependent on the order and resolution. The peaks at $6 \mathrm{~ms}$ are also similar across the different simulations. At later times these peaks decrease, and at $18 \mathrm{~ms}$ the energy distribution is smooth. At $18 \mathrm{~ms}$ and at large $k$, $E_{u^{\prime \prime} u^{\prime \prime}}(k, t)$ from the WENO3C simulation is larger than the spectra from the WENO9 simulations due to aliasing (which causes the energy to accumulate at large wavenumbers as the Nyquist wavenumber $\pi / \Delta x$ is approached). By contrast, the spanwise turbulent kinetic energy spectrum $E_{v^{\prime \prime} v^{\prime \prime}}(k, t)$ is more sensitive to the order and resolution. Higher-order higher-resolution simulations exhibit increased energy content in the intermediate scales. In addition, the WENO5F spectra lie on top of those from the WENO9M simulation, which is consistent with the fact that approximately doubling the order and the resolution yield very similar flow features. The spectra become smoother at later times. Also shown is the turbulent kinetic energy spectrum $E(k, t)=E_{u^{\prime \prime} u^{\prime \prime}}(k, t)+E_{v^{\prime \prime} v^{\prime \prime}}(k, t)$, the behavior of which is dominated by the streamwise component.

The turbulent enstrophy spectrum $E_{\omega^{\prime \prime} \omega^{\prime \prime}}(k, t)$ shown in Fig. 13 is much more sensitive to the order and resolution than $E(k, t)$. In particular, as the order and the resolution increase, the turbulent enstrophy content of all scales increases. The turbulent enstrophy spectrum from the WENO9F simulation 
$6 \mathrm{~ms}$
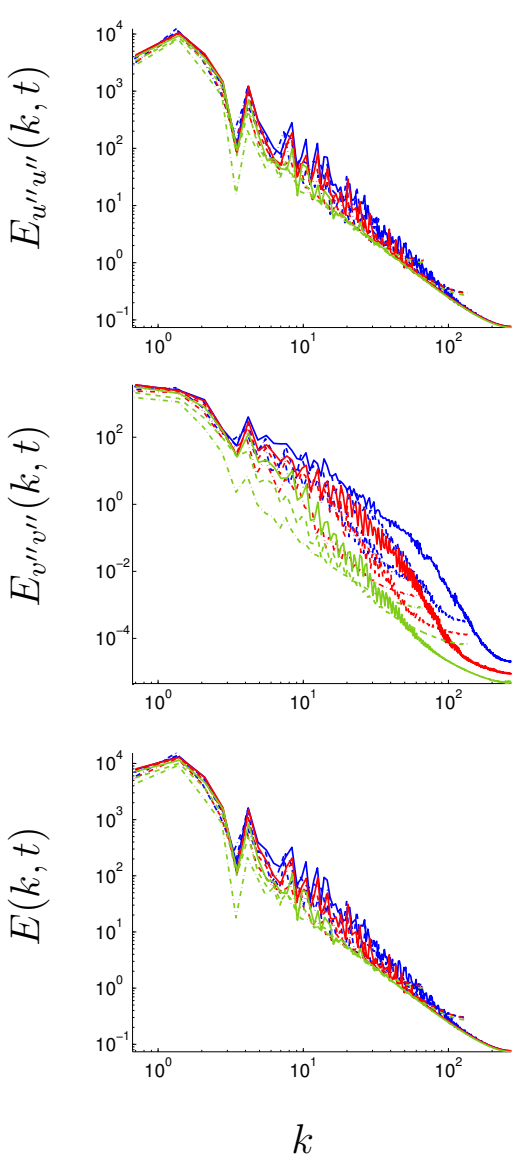

$7 \mathrm{~ms}$
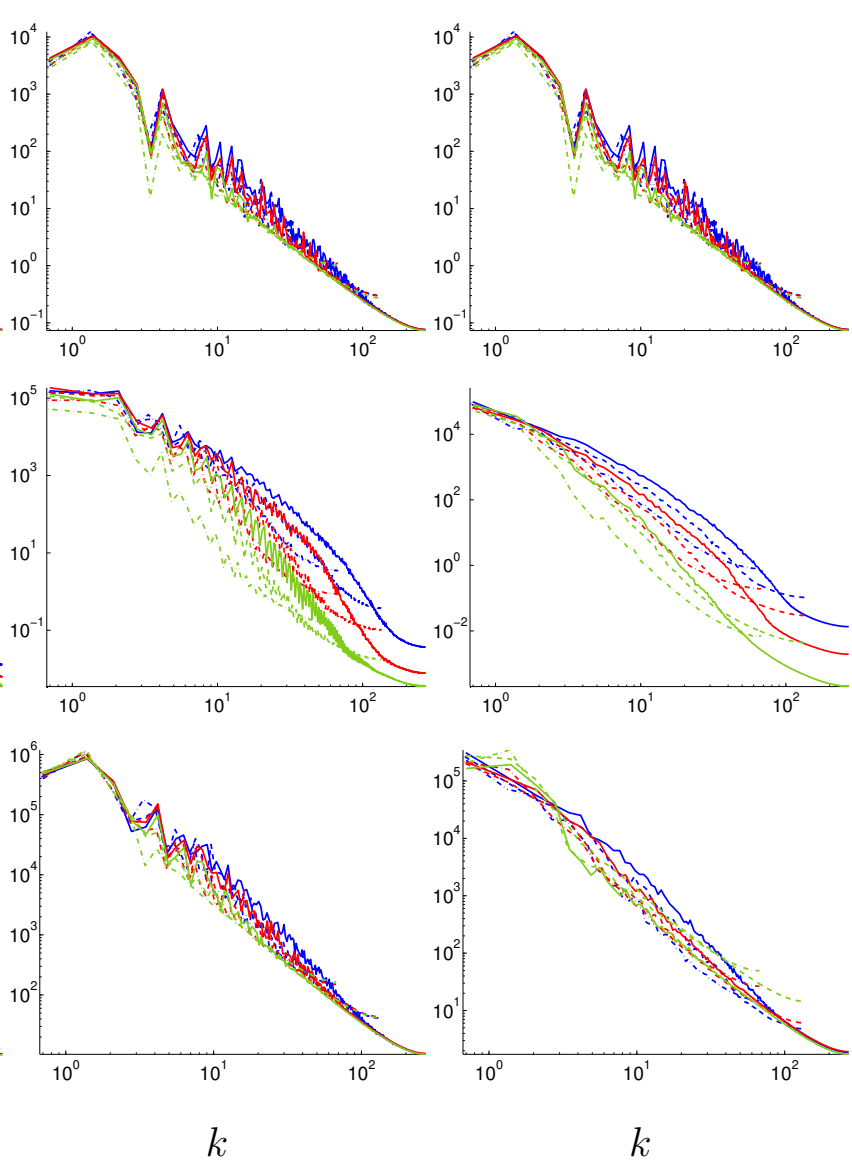

Fig. 12. Comparison of the streamwise and spanwise turbulent kinetic energy spectra $E_{u^{\prime \prime} u^{\prime \prime}}(k, t)$ and $E_{v^{\prime \prime} v^{\prime \prime}}(k, t)$ and turbulent kinetic energy spectrum $E(k, t)$ at 6,7 and $18 \mathrm{~ms}$ when the order and resolution are varied. See Fig. 7 for the legend.

differs from that corresponding to the WENO3C simulation by an order of magnitude in the large scales and by several orders of magnitude in the intermediate and small scales. As shown in Fig. 4, the vorticity corresponding to higher orders and higher resolutions increases in magnitude and is more localized, resulting in the increased values of the spectra. At $18 \mathrm{~ms}$, all of the oscillatory modes have been damped out and the spectra begin to decay. The lower-order lower-resolution spectra are much steeper than those at higher order and resolution. As the vorticity is constructed from the difference of two velocity gradients, $\partial u / \partial y$ and $\partial v / \partial x$, its large wavenumber modes are more sensitive to numerical damping than are primitive fields such as the velocity: this sensitivity is amplified quadratically, as the enstrophy is proportional to the square of the vorticity.

The density variance spectra in Fig. 13 are similar at small wavenumbers. A steep decrease occurs at progressively larger wavenumbers as the order and resolution are increased. This is particularly evident at $18 \mathrm{~ms}$ when all oscil- 

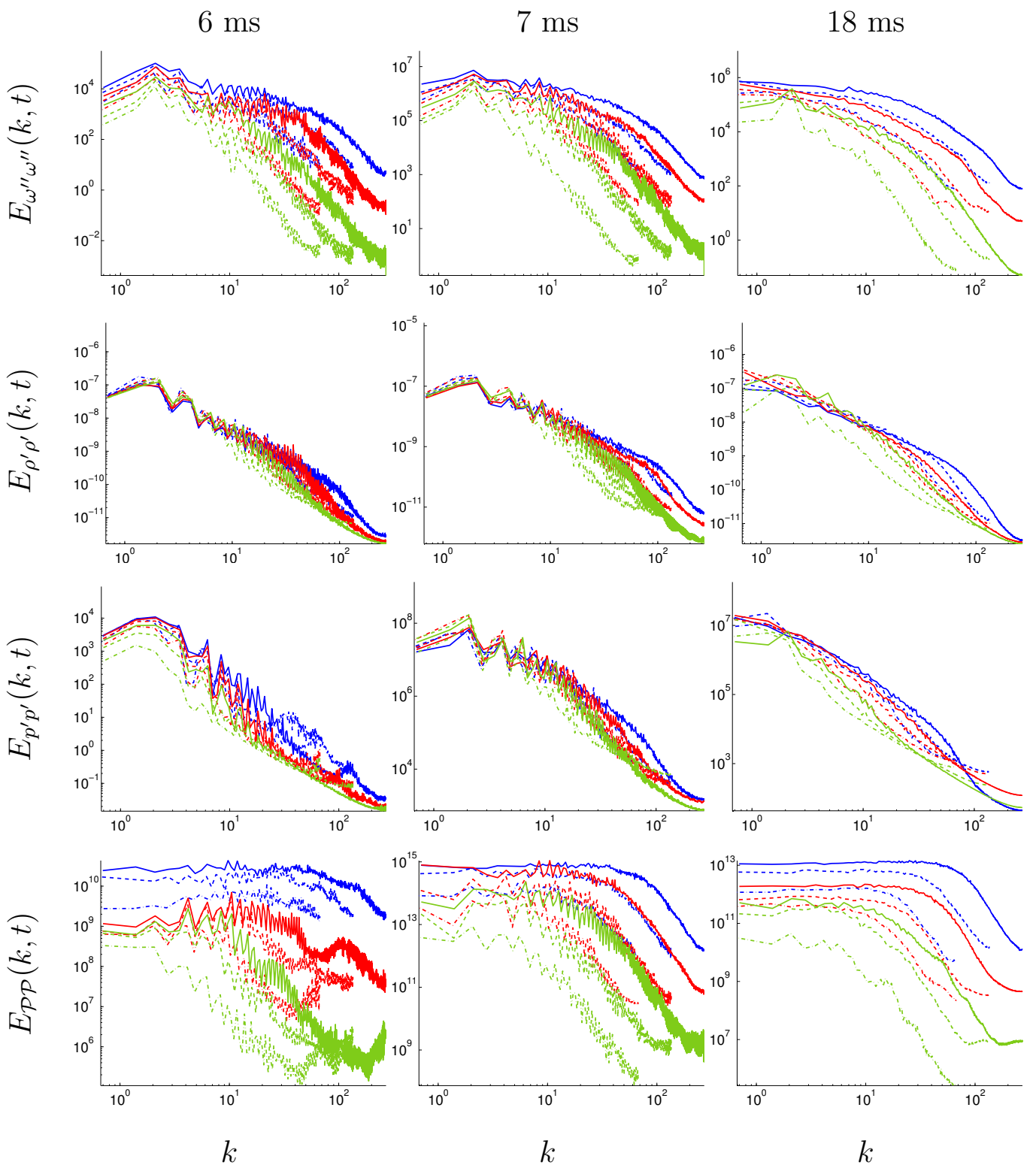

Fig. 13. Comparison of the turbulent enstrophy spectrum $E_{\omega^{\prime \prime} \omega^{\prime \prime}}(k, t)$, density variance spectrum $E_{\rho^{\prime} \rho^{\prime}}(k, t)$, pressure variance spectrum $E_{p^{\prime} p^{\prime}}(k, t)$, and baroclinic vorticity production variance spectrum $E_{\mathcal{P P}}(k, t)$ at 6,7 and $18 \mathrm{~ms}$ when the order and resolution are varied. See Fig. 7 for the legend.

latory modes have been damped out. By contrast, the pressure variance spectrum $E_{p^{\prime} p^{\prime}}(k, t)$ in Fig. 13 exhibits differences even in the small-wavenumber range, with higher order and resolution resulting in increased values. The steep decline in the intermediate- to large-wavenumber spectra can be related to the numerical dissipation, which smooths out fluctuations at large $k$. The lower-order lower-resolution spectra are steeper than the higher-order higherresolution spectra. 
Finally, the baroclinic vorticity production variance spectra $E_{\mathcal{P} P}(k, t)$ in Fig. 13 are nearly uniformly distributed across the scales, as indicated by the nearly constant values over a large range of $k$. This is followed by a rapid decay. Spectra from higher-order higher-resolution simulations remain nearly constant over a larger extent of scales, and are larger in magnitude. By contrast, the spectra from lower-order lower-resolution simulations decay rapidly with increasing $k$, and have smaller values. These results are consistent with the vorticity and baroclinic vorticity production evolution shown in Fig. 6, where $\mathcal{P}(x, y, t)$ from higher-order high-resolution simulations exhibit fragmentation into regions of large positive and negative values. This, in turn, generates vorticity of opposite sign on the interface which contributes to mixing and to the generation of a complex mixing layer. Therefore, increased baroclinic vorticity production in higher-order higher-resolution simulations is correlated with increased mixing and proliferation of complex structures within the layer. As the baroclinic vorticity production variance is proportional to the square of the vector cross product of the density and pressure gradients, the large wavenumber modes of its spectrum are highly sensitive to numerical damping.

In summary, the difference in the dynamics of the small-scales, as well as the energy distribution across scales, was investigated using several energy spectra exhibiting different degrees of sensitivity to the order and resolution. The differences are apparent at all times and become larger at late times, after oscillations have been damped out. The turbulent kinetic energy spectrum $E(k, t)$ exhibits little sensitivity to the order and resolution, whereas the turbulent enstrophy spectrum $E_{\omega^{\prime \prime} \omega^{\prime \prime}}(k, t)$ exhibits significantly more sensitivity, with higher-order lower-resolution simulations having larger values over a broader range of scales. The density variance spectrum $E_{\rho^{\prime} \rho^{\prime}}(k, t)$ also exhibits sensitivity to the order and resolution, particularly at large $k$. The pressure variance spectrum $E_{p^{\prime} p^{\prime}}(k, t)$ exhibits increased sensitivity to order and resolution, both at small and large $k$. Higher-order higher-resolution simulations exhibit baroclinic vorticity production variance spectra $E_{\mathcal{P P}}(k, t)$ that remain large over a larger extent of scales, and that are larger in magnitude. By contrast, lower-order lower-resolution simulations have strongly decaying spectra with smaller values. The WENO9F density variance and pressure variance spectra have an apparent inertial subrange over slightly more than a decade and over approximately one decade in wavenumbers, respectively. The truncation of the density and pressure variance spectra results from the numerical dissipation, which rapidly damps the small-scale fluctuations in the flow. 


\subsection{The statistics}

The effects of order and resolution on the statistics

$$
E_{\phi \phi}(t)=\int_{0}^{k_{\max }} E_{\phi \phi}(k, t) \mathrm{d} k,
$$

including the streamwise and spanwise turbulent kinetic energy and the total turbulent kinetic energy are shown in Fig. 14 as a function of time. Figure 15 shows the turbulent enstrophy, the density and pressure variance, and the baroclinic vorticity production variance as a function of time.

The streamwise turbulent kinetic energy $E_{u^{\prime \prime} u^{\prime \prime}}(t)=\widetilde{u^{\prime \prime 2}} / 2$ decreases prior to reshock, corresponding to the evolution of the roll-up on the spike. As the order and resolution are increased, larger values of energy are obtained. Reshock causes a rapid decrease, followed by a much slower decrease, with all of the simulations giving similar values. Following the arrival of the reflected rarefaction wave at $\approx 11 \mathrm{~ms}$, the lower-order higher-resolution simulations exhibit increased energy. By contrast, the spanwise turbulent kinetic energy $E_{v^{\prime \prime} v^{\prime \prime}}(t)=\widetilde{v^{\prime \prime 2}} / 2$ exhibits an increase prior to reshock, corresponding to the development of the roll-up. The increase is more pronounced in the higher-order higher-resolution simulations as the roll-up develops complex structures. At reshock, the energy increases rapidly and then decays. The decay is faster for the lower-order lower-resolution simulations as energy is not transfered to the spanwise components by the waves. The turbulent kinetic energy $E(t)=\left(\widetilde{u^{\prime \prime 2}}+\widetilde{v^{\prime \prime 2}}\right) / 2$ behaves similarly to its dominant streamwise component $E_{u^{\prime \prime} u^{\prime \prime}}(t)$.

The turbulent enstrophy $E_{\omega^{\prime \prime} \omega^{\prime \prime}}(t)=\widetilde{\omega^{\prime \prime 2}} / 2$ in Fig. 15 exhibits significant sensitivity to the order and resolution. The turbulent enstrophy decreases prior to reshock, with the decrease faster for the lower-order lower-resolution simulations. The higher-order higher-resolution simulations exhibit a smaller decrease, as additional vorticity is deposited in the roll-up by the baroclinic production, which creates the vortex bilayers in Fig. 6. Reshock causes a rapid increase, followed by a steady decrease. Large differences are also observed following reshock as the rapid increase in $E_{\omega^{\prime \prime} \omega^{\prime \prime}}(t)$ caused by reshock is smaller for the lower-order lower-resolution simulations. The turbulent enstrophy is proportional to the square of the fluctuating vorticity. As vorticity fluctuations are responsible for the dissipation of kinetic energy and the transfer of energy to smaller scales, a larger enstrophy from higher-order higher-resolution simulations is associated with the presence of additional small-scale structures.

The density variance $E_{\rho^{\prime} \rho^{\prime}}(t)=\overline{\rho^{\prime 2}} / 2$ in Fig. 15 is sensitive to the order and resolution, particularly at late times. Prior to reshock, lower-order lowerresolution simulations have smaller values, consistent with the suppression of 


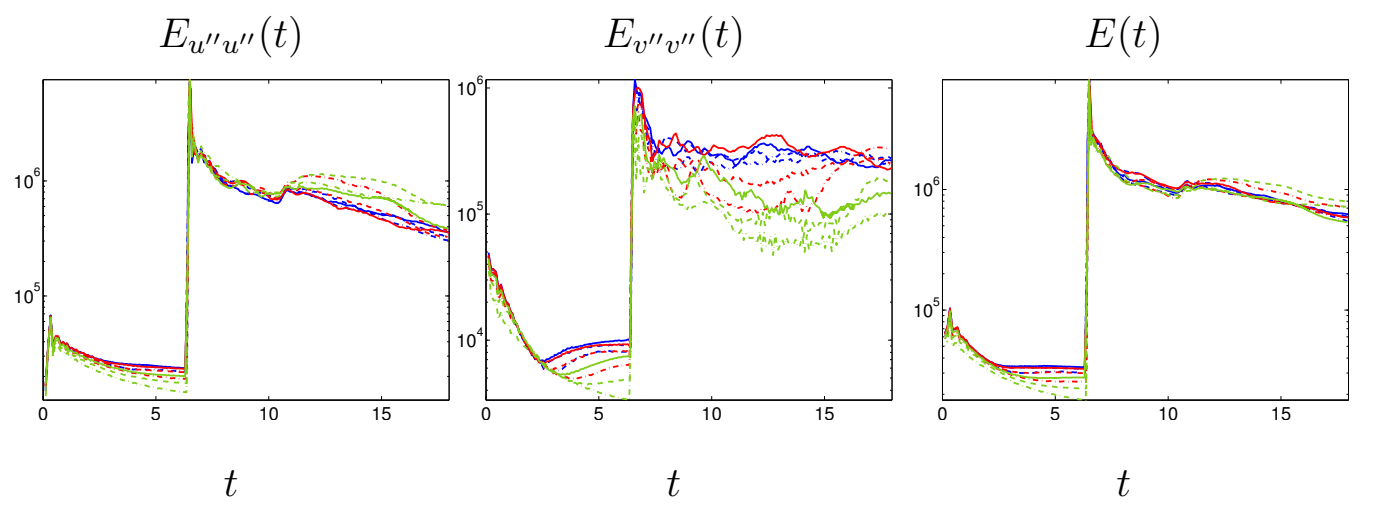

Fig. 14. Comparison of the time-evolution of the streamwise turbulent kinetic energy, spanwise turbulent kinetic energy and total kinetic energy, $E_{u^{\prime \prime} u^{\prime \prime}}(t), E_{v^{\prime \prime} v^{\prime \prime}}(t)$ and $E(t)$, when the order and resolution are varied. See Fig. 7 for the legend.

small scales by increased numerical dissipation. After reshock, a rapid increase is followed by a slow decrease, which becomes more pronounced following the arrival of the reflected rarefaction at $\approx 11 \mathrm{~ms}$. The arrival of the reflected rarefaction amplifies the difference in $E_{\rho^{\prime} \rho^{\prime}}(t)$ across orders. The density variance from the WENO3C and WENO3M simulations is rapidly damped, resulting in a rapid decrease. The pressure variance $E_{p^{\prime} p^{\prime}}(t)$ exhibits little sensitivity to the order and resolution, prior to and following reshock, and at late times.

The baroclinic vorticity production variance $E_{\mathcal{P P}}(t)$ in Fig. 15 is highly sensitive to the order and resolution. This quantity increases prior to reshock in the WENO9M and WENO9F simulations and decreases in all of the other simulations. The increase in the WENO9 simulations is due to the secondary baroclinic vorticity deposition in the roll-up, resulting in the formation of complex structures shown in Fig. 5. These structures are not captured by the other cases. Reshock strongly increases $E_{\mathcal{P P}}(t)$, which is followed by a decay. The arrival of the reflected rarefaction at $\approx 11 \mathrm{~ms}$ further increases $E_{\mathcal{P} \mathcal{P}}(t)$. Following reshock, $E_{\mathcal{P P}}(t)$ from the WENO5F and WENO9M simulations is very similar. A similar relationship exists between higher-order higher-resolution results for the other cases. Prior to reshock, a distinct difference is found between the WENO5 and WENO9 simulations.

In summary, the statistics also exhibited different degrees of sensitivity to the order and resolution, similar to those exhibited by the corresponding energy spectra. The turbulent kinetic energy $E(t)$ exhibited small differences prior to and following reshock, with the differences prior to reshock due to the complex structure observed in the roll-up. A more significant difference was observed in the spanwise turbulent kinetic energy $E_{v^{\prime \prime} v^{\prime \prime}}(t)$ prior to and following reshock due to the spanwise fluctuations excited by the roll-up and the complex structures in the flow. The turbulent enstrophy $E_{\omega^{\prime \prime} \omega^{\prime \prime}}(t)$ and baroclinic vorticity production variance $E_{\mathcal{P} \mathcal{P}}(t)$ exhibited significant variation over all evolution times. The density variance and pressure variance, $E_{\rho^{\prime} \rho^{\prime}}(t)$ and $E_{p^{\prime} p^{\prime}}(t)$, also 

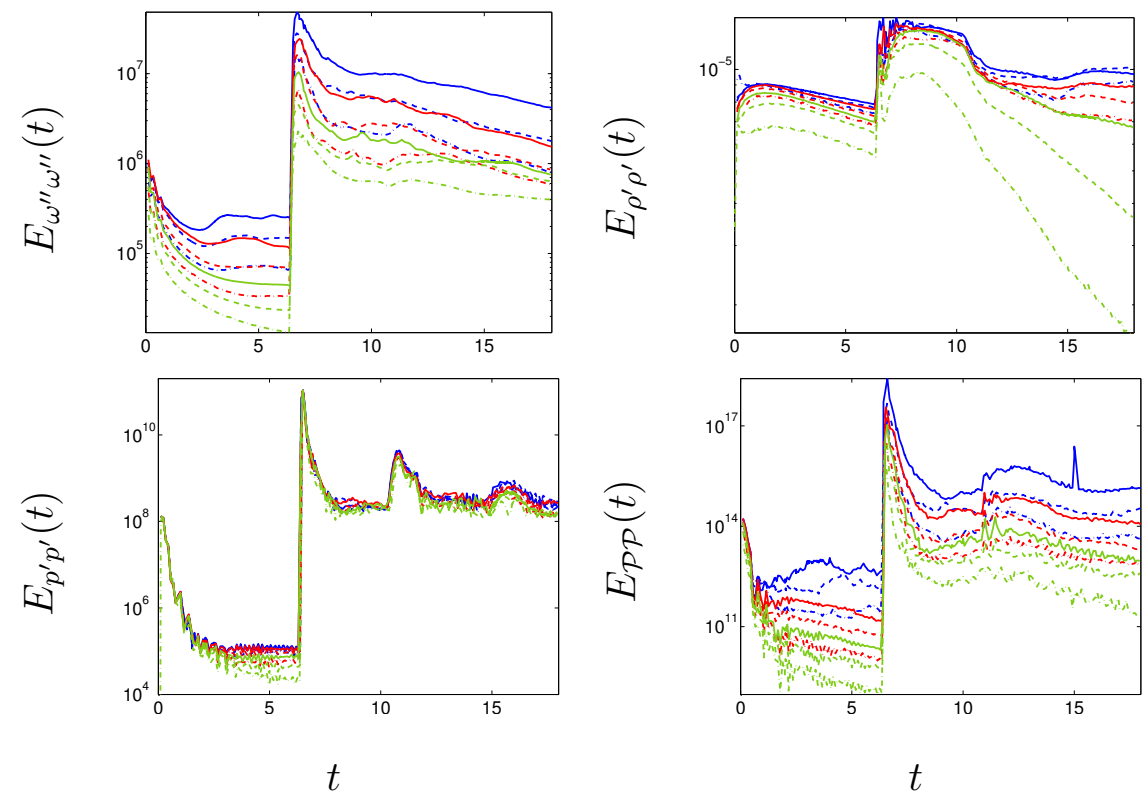

Fig. 15. Comparison of the time-evolution of the turbulent enstrophy, density variance, pressure variance and baroclinic vorticity production variance, $E_{\omega^{\prime \prime} \omega^{\prime \prime}}(t)$, $E_{\rho^{\prime} \rho^{\prime}}(t), E_{p^{\prime} p^{\prime}}(t)$ and $E_{\mathcal{P} \mathcal{P}}(t)$, when the order and resolution are varied. See Fig. 7 for the legend.

exhibited variations prior to and following reshock.

\subsection{The probability distribution functions}

A novel quantitative measure of the effects of order and resolution on primitive, fluctuating and derived scalar fields represented on the discrete computational grid is provided by the probability distribution. The probability with which a particular value of a scalar field $\phi(x, y, t)$ occurs at a particular time during the evolution of the instability can be quantified using the probability distribution function (PDF) $P(\phi, t)$. Consider the field $\phi(x, y, t)$ within the mixing layer $(x, y) \in\left[\ell_{s}, \ell_{b}\right] \times\left[0, L_{y}\right]$ discretized by $N_{x} \times N_{y}$ points. Divide the range $[\min (\phi), \max (\phi)]$ into $M=\sqrt{N_{x} N_{y}} / 2$ (an integer) equal bins $\Delta \phi \equiv[\max (\phi)-\min (\phi)] / M$. Each discrete value of $\phi\left(x_{i}, y_{j}, t\right)$ is distributed in the bins, yielding the frequency $N_{k}$, so that the PDF is defined as

$$
P_{k}(\phi, t)=\frac{N_{k}}{\Delta \phi N_{x} N_{y}}
$$

satisfying $\sum_{k=1}^{M} P_{k}(\phi, t) \Delta \phi=1$ (or $\int_{-\infty}^{\infty} P(\phi, t) \mathrm{d} \phi=1$ in the continuum limit).

At $6 \mathrm{~ms}$, the density PDF $P(\rho)$ in Fig. 16 has two peaks corresponding to the density of the air(acetone) (left peak) and of the $\mathrm{SF}_{6}$ (right peak). The values between the peaks provide a measure of the 'molecular mixing'. As the order 
$6 \mathrm{~ms}$

$7 \mathrm{~ms}$

$18 \mathrm{~ms}$
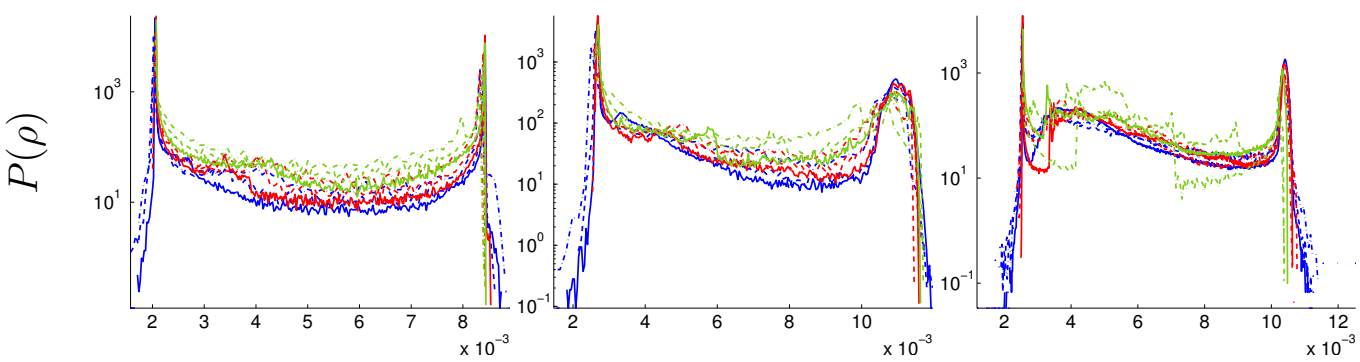

$\rho$

$\rho$

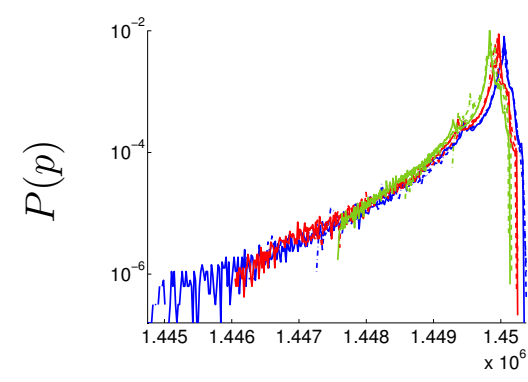

$p$ $\rho$

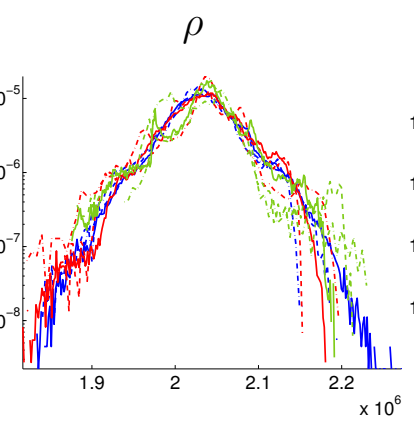

$p$

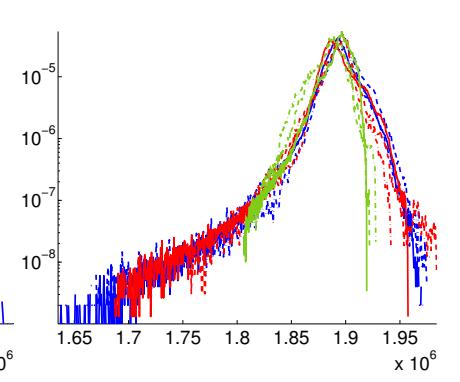

$p$

Fig. 16. Comparison of the PDFs of the density and pressure $P(\rho)$ and $P(p)$ at 6 , 7 and $18 \mathrm{~ms}$ when the order and the grid resolution are varied. See Fig. 7 for the legend.

and resolution are increased, the magnitude of the PDF between the peaks decreases as expected, with a corresponding decrease in numerical dissipation (and therefore of numerically-induced mixing). At $7 \mathrm{~ms}$, the PDF exhibits a smoother peak in the $\mathrm{SF}_{6}$ region, corresponding to the reflected rarefaction creating a wider range of scales, whereas the peak in the air(acetone) region is compressed by the passage of the transmitted shock, creating a narrower range of scales. The PDF at $7 \mathrm{~ms}$ exhibits increased sensitivity to the order and resolution. By contrast, $P(\rho)$ at $18 \mathrm{~ms}$ shows decreased sensitivity for sufficiently high order and resolution. At $18 \mathrm{~ms}$, the layer is nearly uniformly mixed. The pressure PDF $P(p)$ at 6 ms has a single peak, corresponding to the pressure following shock passage. At $7 \mathrm{~ms}$, the pressure peak is broadened by the reflected rarefaction, which further narrows at $18 \mathrm{~ms}$. As the order is increased, the higher-order higher-resolution simulations exhibit PDFs with longer tails, indicating the presence of additional smaller scales.

Figure 17 shows the PDFs of the components of the density gradient $P(\partial \rho / \partial x)$ and $P(\partial \rho / \partial y)$ and of the pressure gradient $P(\partial p / \partial x)$ and $P(\partial p / \partial y)$, which exhibit increased sensitivity to the order and resolution. Higher-order higherresolution simulations exhibit a wider range of scales, reflected in broader values of the PDFs. It is important to appreciate that the components of the density and pressure gradients form the baroclinic vorticity production $\mathcal{P}$ (the 
$6 \mathrm{~ms}$

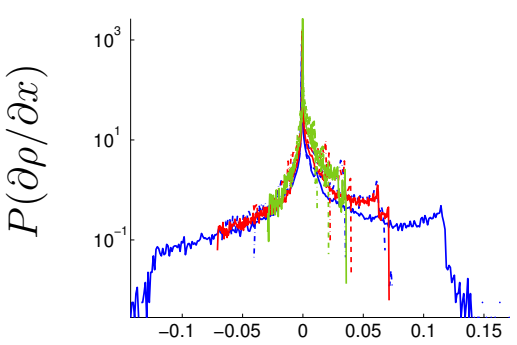

$\partial \rho / \partial x$

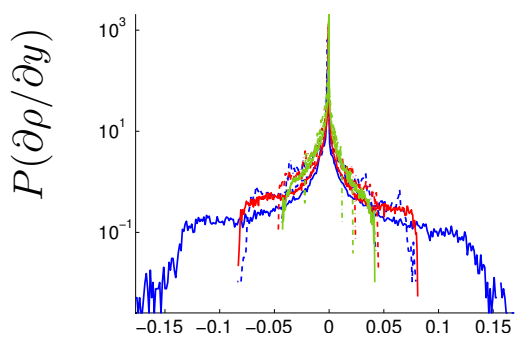

$\partial \rho / \partial y$

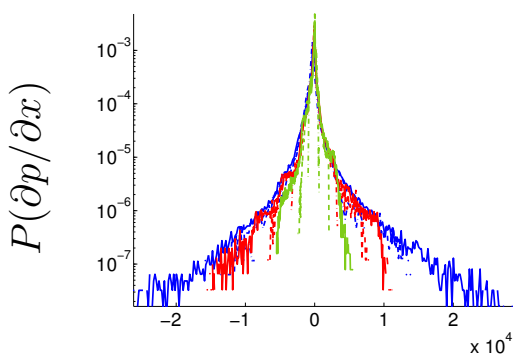

$\partial p / \partial x$

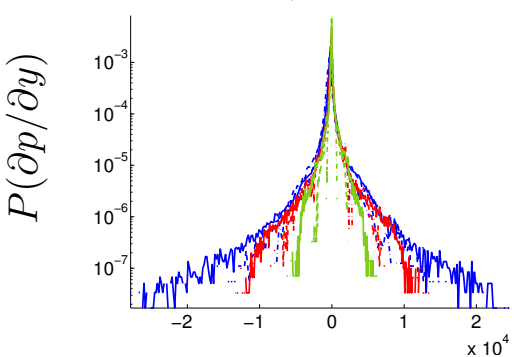

$\partial p / \partial y$
$7 \mathrm{~ms}$

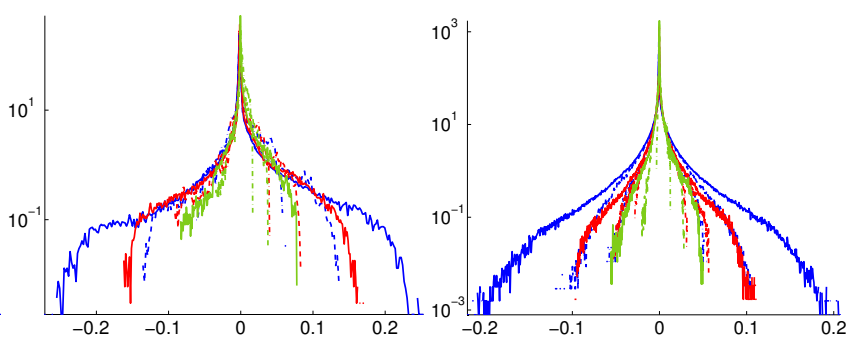

$\partial \rho / \partial x$
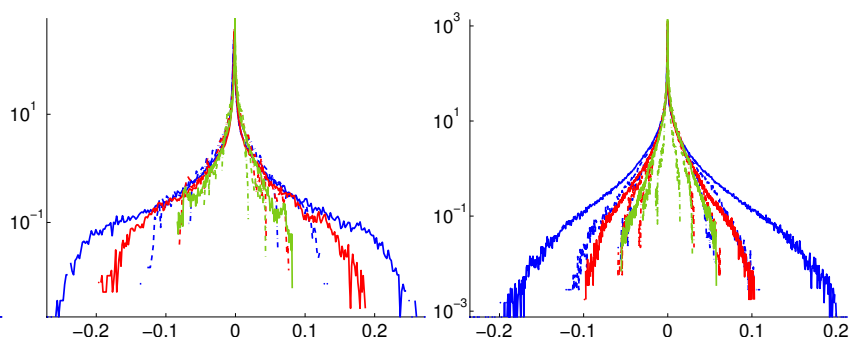

$\partial \rho / \partial y$

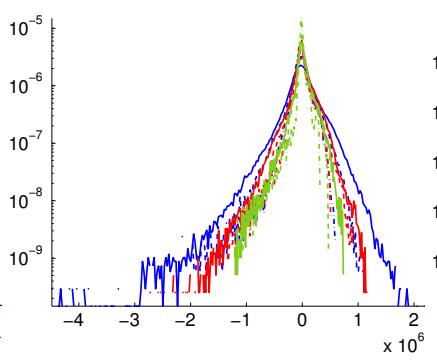

$\partial p / \partial x$

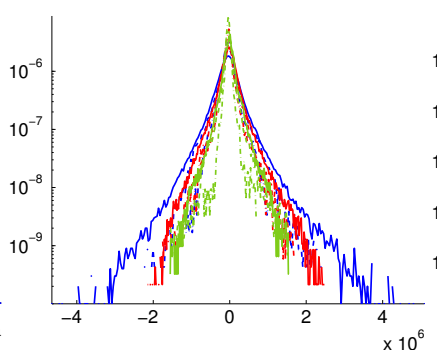

$\partial p / \partial y$ $\partial \rho / \partial y$

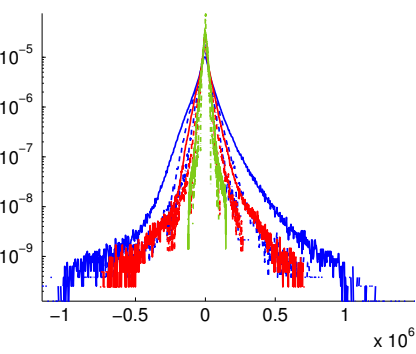

$\partial p / \partial x$

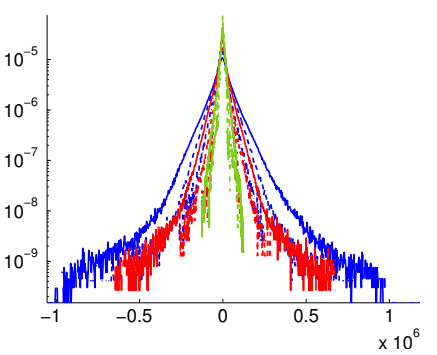

$\partial p / \partial y$

Fig. 17. Comparison of the PDF of the components of the density gradient $P(\partial \rho / \partial x)$ and $P(\partial \rho / \partial y)$ and pressure gradient $P(\partial p / \partial x)$ and $P(\partial p / \partial y)$ at 6,7 and $18 \mathrm{~ms}$ when the order and resolution are varied. See Fig. 7 for the legend.

PDF of which is shown in Fig. 19): the PDFs of the gradients indicate that higher-order higher-resolution simulations yield larger values of the baroclinic vorticity production.

At $6 \mathrm{~ms}$ the PDF of the streamwise velocity fluctuation $P\left(u^{\prime \prime}\right)$ in Fig. 18 ex- 

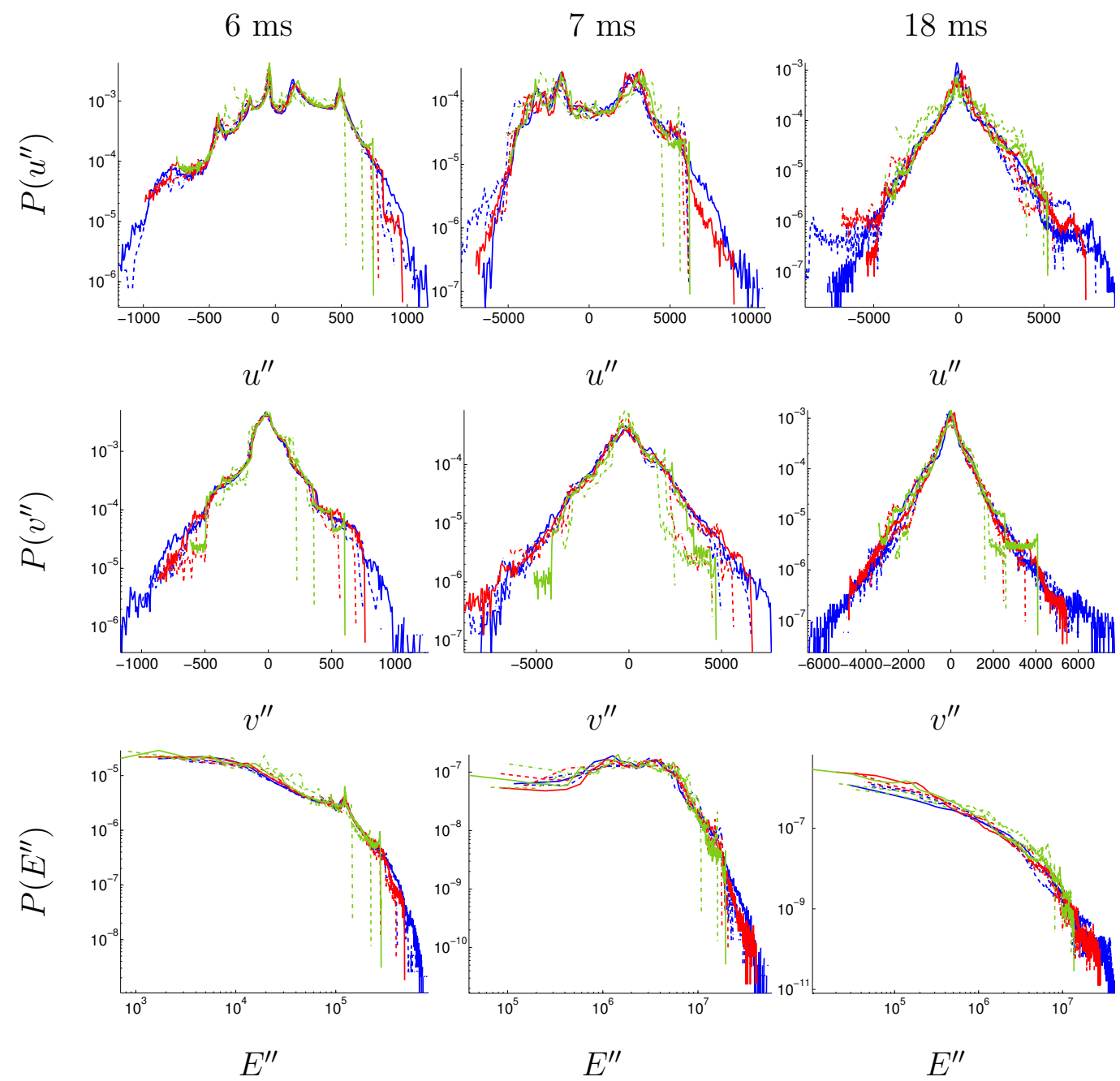

Fig. 18. Comparison of the PDFs of the streamwise and spanwise velocity fluctuation $P\left(u^{\prime \prime}\right)$ and $P\left(v^{\prime \prime}\right)$ and fluctuating kinetic energy $P\left(E^{\prime \prime}\right)$ at 6,7 and $18 \mathrm{~ms}$ when the order and resolution are varied. See Fig. 7 for the legend.

hibits several peaks corresponding to the different values in the roll-up regions. At $7 \mathrm{~ms}$, two peaks corresponding to the rapid expansion (in both directions) of the mixing layer form. At late times, a single symmetric peak forms, corresponding to a decaying mixing layer. All of the simulations capture the shapes and the values of the peaks. However, higher-order higher-resolution simulations exhibit wider PDFs, corresponding to larger (positive and negative) values of the fluctuations. The PDF of the spanwise velocity fluctuation $P\left(v^{\prime \prime}\right)$ exhibits a single peak at all times. The peak is slightly asymmetric at 6 and $7 \mathrm{~ms}$, corresponding to the presence of the roll-ups, and becomes symmetric at $18 \mathrm{~ms}$. As in the case of the streamwise component, all of the simulations capture the peak and have similar widths. However, higher-order higher-resolution simulations exhibit additional scales at larger values of the fluctuations. Finally, the PDF of the fluctuating kinetic energy per unit mass $P\left(E^{\prime \prime}\right)$, where $E^{\prime \prime}=\left(u^{\prime \prime 2}+v^{\prime \prime 2}\right) / 2$, indicates that all simulations have similar 

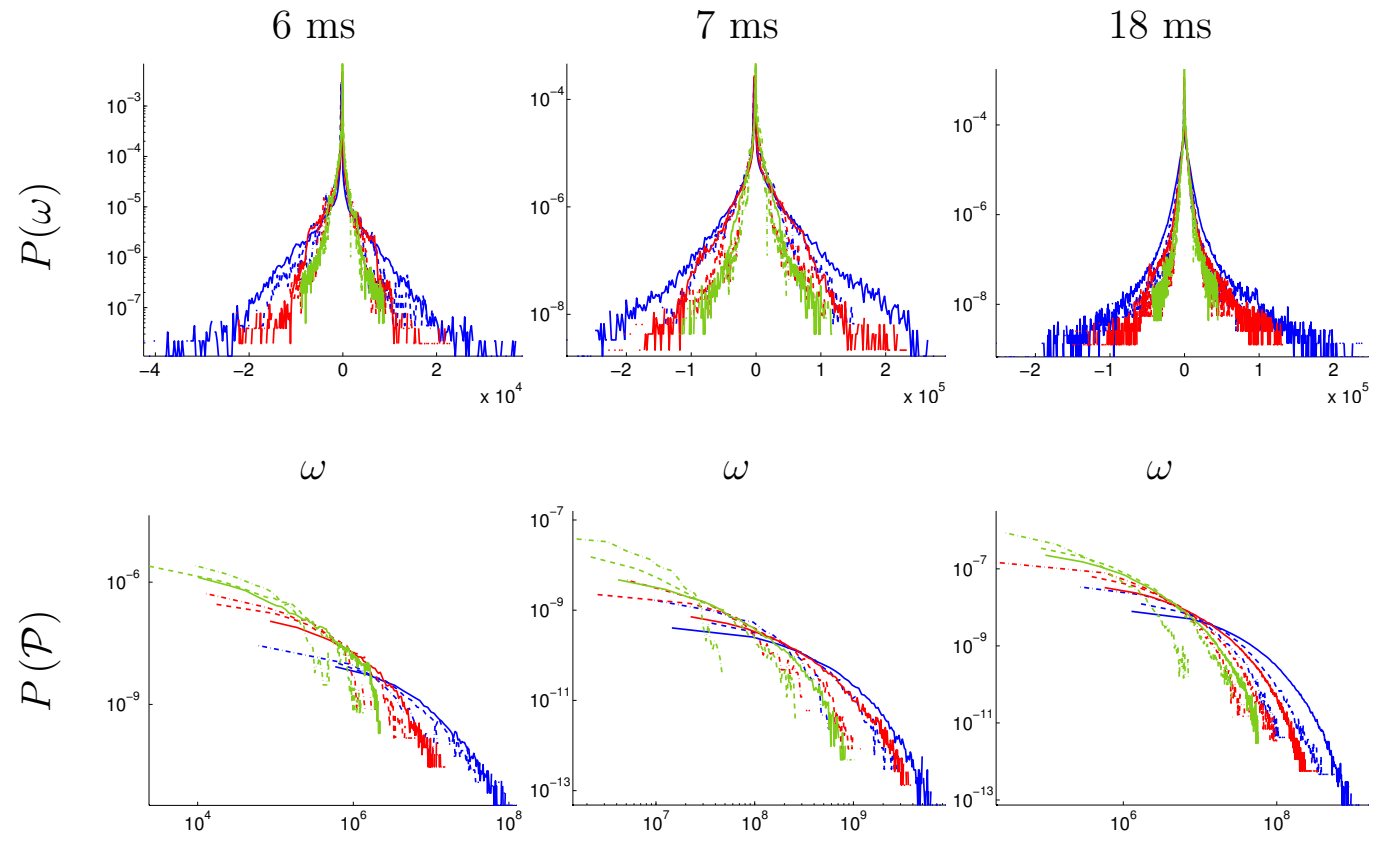

$\mathcal{P}$

$\mathcal{P}$

$\mathcal{P}$

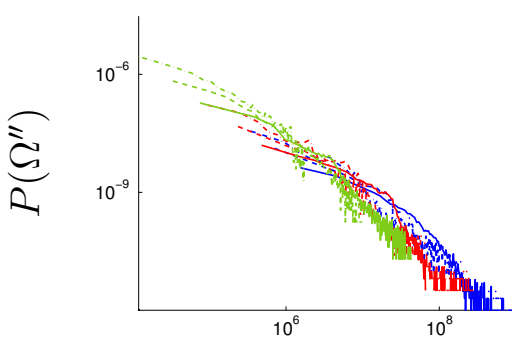

$\Omega^{\prime \prime}$
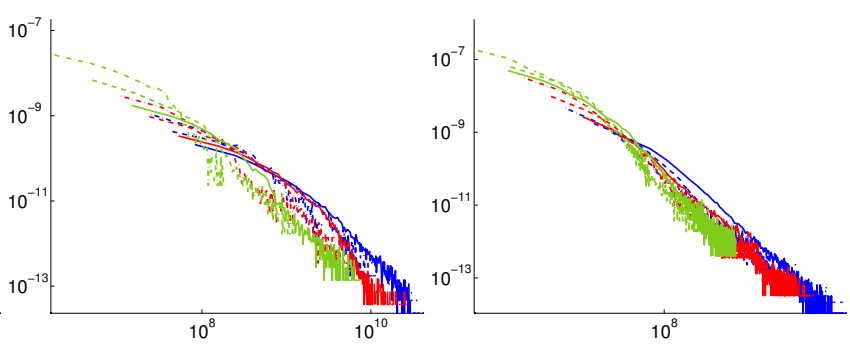

$\Omega^{\prime \prime}$

$\Omega^{\prime \prime}$

Fig. 19. Comparison of the PDFs of the vorticity $P(\omega)$, baroclinic vorticity production $P(\mathcal{P})$, and fluctuating enstrophy $P\left(\Omega^{\prime \prime}\right)$ at 6,7 and $18 \mathrm{~ms}$ when the order and resolution are varied. See Fig. 7 for the legend.

distributions at small values. As the values increase, the lower-order lowerresolution simulations exhibit a sudden decrease, corresponding to numerically damped smaller-scale fluctuations.

The PDF of the vorticity $P(\omega)$ in Fig. 19 exhibits a single symmetric peak at all times. Higher-order higher-resolution simulations exhibit broader peaks, consistent with more localized and larger vorticity. The peak is particularly broad following reshock at $7 \mathrm{~ms}$. Reshock deposits vorticity on the interface via the baroclinic vorticity production mechanism. As shown in Fig. 5, the higherorder simulations exhibit additional structures on the roll-up that contribute to increased vorticity deposition on the interface. The differences in the PDFs among orders and resolutions decrease at late times. The PDF of the baroclinic vorticity production $P(\mathcal{P})$ is shown with the values on the horizontal axis on a logarithmic scale, indicating that lower-order lower-resolution simulations have 
higher probability of the production at smaller values. By contrast, higherorder higher-resolution simulations exhibit larger values of the PDFs, which can be explained by the broader PDFs of the density and pressure gradient components shown in Fig. 17. The PDFs of the fluctuating enstrophy per unit mass $P\left(\Omega^{\prime \prime}\right)$, where $\Omega^{\prime \prime}=\omega^{\prime \prime 2} / 2$, from lower-order lower-resolution simulations exhibit a higher probability of smaller values of the enstrophy, whereas higherorder higher-resolution simulations exhibit a broader range of values.

In summary, the probability distribution functions (PDFs) were considered to investigate the distribution of values of various primitive, fluctuating and derived scalar fields in the flow, and it was found that while the different simulations may give similar predictions of mean values, quantities depending on smaller-scale structures or with extreme values will differ, i.e., the tails of the PDFs corresponding to the extreme values are broader or narrower, depending on the relative amount of numerical dissipation. Specifically, when fluctuating fields are considered, the higher-order higher-resolution simulations exhibit broader PDFs corresponding to a wider range of values. Similarly, when gradients of fields are considered, higher-order higher-resolution simulations exhibit wider PDFs corresponding to larger absolute values of the gradients. As a result, when the vorticity or baroclinic vorticity production are considered (which depend on the gradients of the velocity components, and on the gradients of the density and pressure fields, respectively), the higher-order lower-resolution simulations yield an increased range of values of these fields.

\section{Quantification of numerical dissipation: turbulent kinetic energy and enstrophy production/dissipation rate, numerical Reynolds numbers and numerical viscosity}

In this section the dependence of the results shown in Secs. 3-5 on the order and resolution is interpreted by computing the (implicit) numerical dissipation of the WENO method. The numerical dissipation associated with each simulation is quantified by the turbulent kinetic energy and enstrophy production/dissipation rates, the numerical Reynolds numbers, and the effective numerical viscosity (dissipation).

\subsection{The numerical turbulent kinetic energy and enstrophy production/dissipation rates}

Here the effective numerical turbulent kinetic energy production/dissipation rate $\widetilde{\epsilon^{\prime \prime}}(t)$ is computed from the simulations using two different expressions. In the case of an Euler simulation of the reshocked Richtmyer-Meshkov in- 


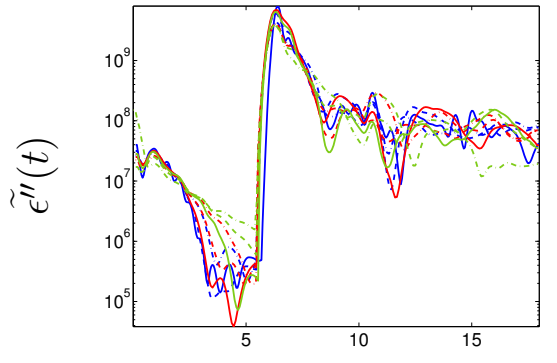

$t$

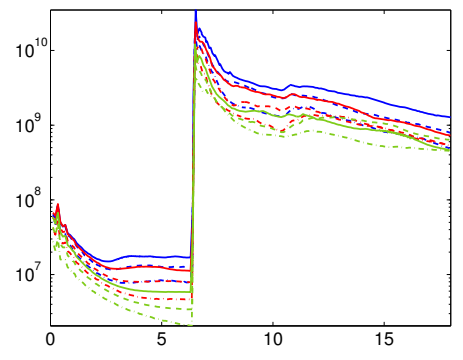

$t$

Fig. 20. Comparison of the time-evolution of the effective turbulent kinetic energy production/dissipation rate $\widetilde{\epsilon^{\prime \prime}}(t)$, computed using Eqs. (17) and (18) (left and right), respectively, when the order and resolution are varied. See Fig. 7 for the legend.

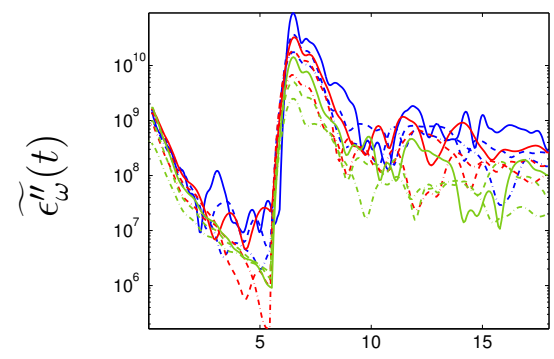

$t$

Fig. 21. Comparison of the time-evolution of the effective turbulent enstrophy production/dissipation rate $\widetilde{\epsilon_{\omega}^{\prime \prime}}(t)$, computed using Eq. (19) when the order and resolution are varied. See Fig. 7 for the legend.

stability, the turbulent kinetic energy is dissipated by numerical mechanisms after the shock has passed through the interface. The interaction of the shock with the interface (or mixing layer) imparts kinetic energy into the flow field (production). As $\widetilde{\epsilon^{\prime \prime}}$ is not uniquely defined in the present simulations, two independent methods for computing this quantity are used and compared.

The first method to quantify $\widetilde{\epsilon^{\prime \prime}}(t)$ over the flow evolution entails computing the negative of the time-rate-of-change of the turbulent kinetic energy (see Fig. 14) [36],

$$
\tilde{\epsilon}^{\prime \prime}(t)=-\frac{\mathrm{d} E(t)}{\mathrm{d} t}
$$

Another estimate of $\tilde{\epsilon}^{\prime \prime}$ is given by turbulent transport phenomenology [37]

$$
\tilde{\epsilon^{\prime \prime}}(t)=E(t) \sqrt{2 E_{\omega^{\prime \prime} \omega^{\prime \prime}}(t)},
$$

where $E_{\omega^{\prime \prime} \omega^{\prime \prime}}(t)=\widetilde{\omega^{\prime \prime 2}} / 2$ is the turbulent enstrophy per unit mass. Both formulations of $\epsilon^{\prime \prime}$ shown in Fig. 20 are qualitatively similar. In particular, $\widetilde{\epsilon^{\prime \prime}}$ peaks following the passage of the initial incident shock and then decreases. Reshock sharply increases $\widetilde{\epsilon^{\prime \prime}}$ by four to five orders of magnitude as reshock 
rapidly deposits energy into the layer. Following reshock, $\widetilde{\epsilon^{\prime \prime}}$ decreases rapidly. The turbulent kinetic energy production/dissipation rate computed using Eq. (17) oscillates as this expression is based on numerically differentiating $E(t)$ (introducing noise). To remedy this, the five-point average of $\widetilde{\epsilon^{\prime \prime}}$ was computed prior to differentiation. The turbulent kinetic energy production/dissipation rate based on Eq. (18) shows that the WENO9F simulation yields the largest value of $\widetilde{\epsilon^{\prime \prime}}$, as the increased kinetic energy of higher-order higher-resolution simulations corresponds to larger values of $\widetilde{\epsilon}^{\prime \prime}$. Similarly, the WENO3C simulation has the smallest value of $\widetilde{\epsilon}^{\prime \prime}$, as the corresponding kinetic energy content is lower.

The effective numerical turbulent enstrophy production/dissipation rate $\widetilde{\epsilon_{\omega}^{\prime \prime}}(t)$ can also be computed from the numerical simulations. As in the case of the turbulent kinetic energy, the turbulent enstrophy is dissipated by numerical mechanisms after the shock has passed through the interface. The interaction of the shock with the interface (or mixing layer) deposits vorticity (circulation) on the interface and, therefore, increases the turbulent enstrophy and its dissipation rate. The negative of the time-rate-of-change of $\widetilde{\epsilon_{\omega}^{\prime \prime}}(t)$ provides a direct measure of the production/dissipation rate:

$$
\widetilde{\epsilon_{\omega}^{\prime \prime}}(t)=-\frac{\mathrm{d} E_{\omega^{\prime \prime} \omega^{\prime \prime}}(t)}{\mathrm{d} t}
$$

The turbulent enstrophy production/dissipation rate $\widetilde{\epsilon_{\omega}^{\prime \prime}}(t)$ in Fig. 21 exhibits a qualitative behavior similar to that of $\widetilde{\epsilon^{\prime \prime}}(t)$ in Fig. 20. A peak is observed following the passage of the initial incident shock, followed by a decrease. Reshock further increases $\widetilde{\epsilon_{\omega}^{\prime \prime}}$ by three to four orders of magnitude, followed by a rapid decrease. The $\widetilde{\epsilon_{\omega}^{\prime \prime}}$ corresponding to the WENO9 simulations are the largest, indicating that the enstrophy is produced and dissipated at a faster rate in higher-order higher-resolution simulations.

In summary, the turbulent kinetic energy production/dissipation rate $\widetilde{\epsilon^{\prime \prime}}(t)$ was computed using the negative of the time-rate-of-change of the kinetic energy and using the product of the turbulent kinetic energy and the turbulent enstrophy, which were shown to provide similar characterizations of the numerical dissipation rate. In particular, higher levels of turbulent kinetic energy production/dissipation are associated with the higher-order high-resolution simulations, as their turbulent kinetic energy content is larger than in the lower-order lower-resolution simulations The turbulent enstrophy production/dissipation rate $\widetilde{\epsilon_{\omega}^{\prime \prime}}(t)$ was computed by taking the negative of the time-rate-of-change of the turbulent enstrophy. Larger values of $\epsilon_{\omega}^{\prime \prime}(t)$ were obtained in higher-order higher-resolution simulations, as the enstrophy content in these simulations is larger; this trend is similar to that of $\tilde{\epsilon}^{\prime \prime}(t)$. 

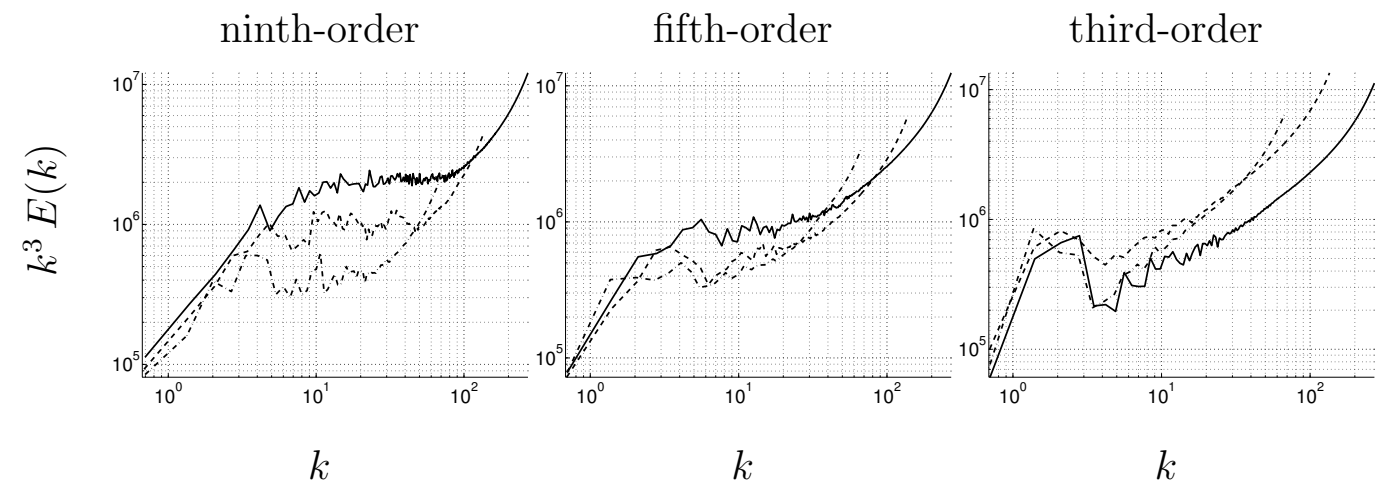

Fig. 22. Comparison of the compensated turbulent kinetic energy spectra $k^{3} E(k)$ at $18 \mathrm{~ms}$ when the order and resolution are varied. Quantities obtained on the fine, medium, and coarse grids are shown using a solid, dashed and dash-dot line, respectively.

\subsection{The numerical Reynolds numbers}

Another quantification of the effects of the numerical dissipation is given by computing a numerical Reynolds number. In the present simulations based on the Euler equations, there is no unique definition of the Reynolds number. Thus, two different definitions are presented and compared. The first definition is that used in three-dimensional blast wave simulations [38]

$$
\operatorname{Re}_{h}(t)=\left[\frac{h(t)}{\Delta x}\right]^{4 / 3},
$$

where $h(t)$ is the mixing layer width and $\Delta x$ is the grid spacing. This Reynolds number is based on the largest scale in the flow given by the mixing layer width $h(t)$. Note that each doubling of the resolution contributes to an increase of $2^{4 / 3} \approx 2.5$ in $\operatorname{Re}_{h}$.

A second definition of the Reynolds number was used in the context of threedimensional two-mode Richtmyer-Meshkov instability simulations [10]:

$$
\operatorname{Re}_{\ell}(t)=\left[\frac{\ell(t)}{\ell_{d}}\right]^{4 / 3},
$$

where

$$
\ell(t)=\frac{3 \pi}{4} \frac{\int_{0}^{k_{\max }} \frac{E(k, t)}{k} \mathrm{~d} k}{\int_{0}^{k_{\max }} E(k, t) \mathrm{d} k}
$$

is the integral length scale [39] and $\ell_{d}=2 \pi / k_{d}$ is the Kolmogorov length scale. The Kolmogorov wavenumber can be estimated as $k_{d}=50 k_{\nu}$ [40], where the dissipation wavenumber $k_{\nu}$ is the value of $k$ at which the turbulent kinetic energy spectrum $E(k, t)$ begins to steepen from the -3 law expected for 

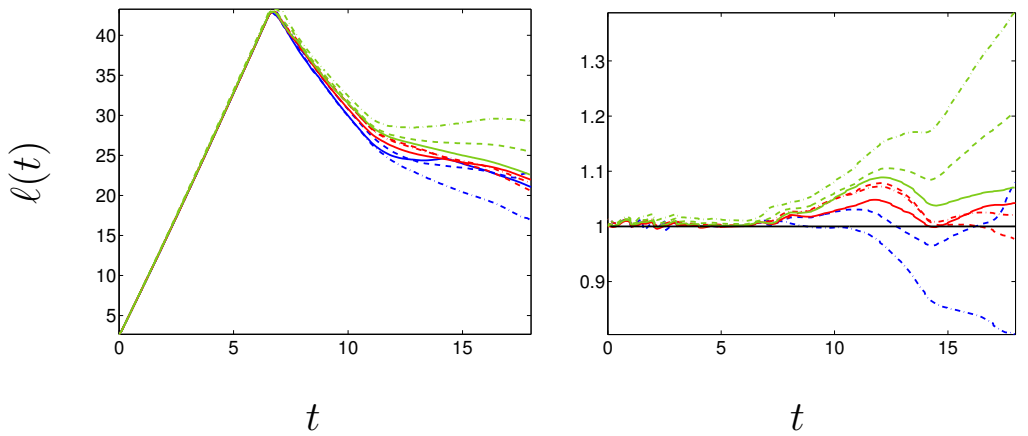

Fig. 23. Comparison of the time-evolution of the integral length scale $\ell(t)$ [see Eq. (22)] when the order and resolution are varied (left). The integral length scales normalized by that from the WENO9F simulation is also shown (right). See Fig. 7 for the legend.

two-dimensional turbulent flows exhibiting an inertial subrange [4,41]. The Reynolds number given by Eq. (21) is based on the integral length scale $\ell(t)$ which is related to the energy content of the largest scales. Thus, the two Reynolds numbers are expected to behave differently as they are based on different length scales. Both Reynolds numbers were used in the context of three-dimensional simulations. However, as the estimates are obtained from evaluating the range of scales along a single spatial direction, these definitions are also appropriate for the present two-dimensional simulations.

First, the values of $k_{\nu}$ are determined by plotting the compensated turbulent kinetic energy spectra $k^{3} E(k)$ shown in Fig. 22, where the WENO9, WENO5 and WENO3 results are shown for each of the grid resolutions considered: $k_{\nu}$ corresponds to the wavenumber where the spectrum is no longer horizontal, which is estimated by visual inspection. The compensated spectra are shown at $18 \mathrm{~ms}$, but it was verified that $k_{\nu}$ changes very little when the spectra are considered at 6,7 and $12 \mathrm{~ms}$ instead.

Prior to reshock, the integral length scale $\ell(t)$ in Fig. 23 increases and is very weakly dependent on the order and resolution. By contrast, following reshock $\ell(t)$ decreases with increased sensitivity to the order and resolution. In particular, higher-order higher-resolution simulations correspond to smaller values of $\ell(t)$. The agreement in the value of $\ell(t)$ prior to reshock indicates similar energy content in the largest scales. Following reshock, the forward cascade of energy from the larger scales to the smaller scales results in smaller values of $\ell(t)$. The forward cascade is more pronounced in higher-order higherresolution simulations, which results in even smaller values of $\ell(t)$. The arrival of the reflected rarefaction at $\approx 11 \mathrm{~ms}$ deposits energy, which slows down the decrease in the integral length scale. However, this process is balanced by the forward cascade process, resulting in increased sensitivity to the order and resolution. Up to the arrival of the reflected rarefaction, the $\ell(t)$ are within $10 \%$ of one another. Note that $\ell(t)>h(t)$ up to reshock. Also, $\ell(t)$ grows 

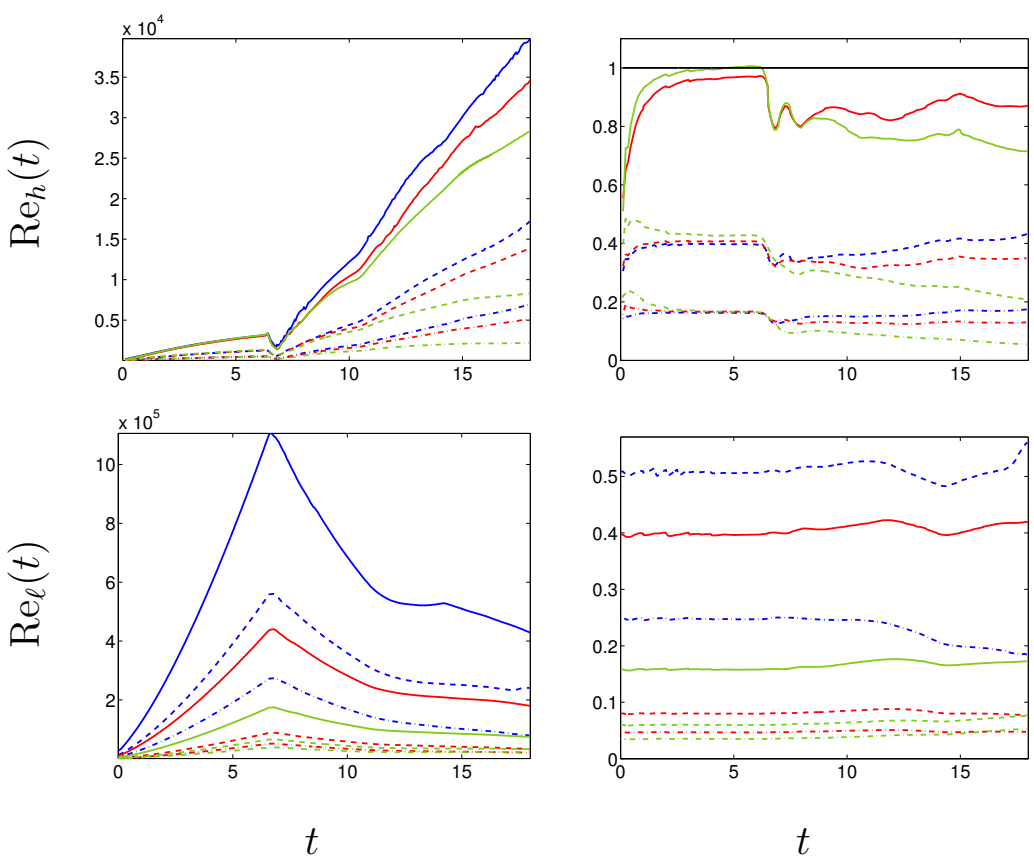

Fig. 24. Comparison of the time-evolution of the numerical Reynolds numbers $\operatorname{Re}_{h}(t)$ [see Eq. (20)] and $\operatorname{Re}_{\ell}(t)$ [see Eq. (21)] when the order and resolution are varied (left column). The numerical Reynolds numbers normalized by that from WENO9F simulation are also shown (right column). See Fig. 7 for the legend.

linearly in time prior to reshock and decays linearly in time after reshock, prior to the arrival of the reflected rarefaction.

Both numerical Reynolds numbers in Fig. 24 increase prior to reshock. However, the $\mathrm{Re}_{h}$ are grouped by the resolution (as shown by the ratio of the Reynolds numbers), while the $\mathrm{Re}_{\ell}$ are grouped by the order and resolution. After reshock, the two Reynolds numbers behave entirely differently. The Reynolds number based on $h(t)$ decreases due to the compression of the mixing layer caused by reshock, while the Reynolds number based on $\ell(t)$ peaks at reshock due to the large energy deposition during reshock. Following reshock, $\mathrm{Re}_{h}$ increases while $\mathrm{Re}_{\ell}$ decreases as the energy cascades from the large to small scales. Thus, the two Reynolds numbers provide entirely different characterizations based on different length scales. Note that $\mathrm{Re}_{\ell}$ peaks at $\sim 1.1 \times 10^{6}$ for the WENO9F simulation, with the peaks from all other simulations considerably lower.

In summary, two numerical Reynolds numbers based on different estimates of the range of length scales resolved in the flow were computed and compared. The first Reynolds number, $\operatorname{Re}_{h}(t)$, is based on the range of scales between the grid resolution $\Delta x$ and the mixing layer width $h(t)$, while the second Reynolds number, $\operatorname{Re}_{\ell}(t)$, is based on the range of scales between the integral length scale $\ell(t)$ and the numerical analog of the Kolmogorov dissipation length scale $\ell_{d}$. The former Reynolds number is associated with the largest scales present 

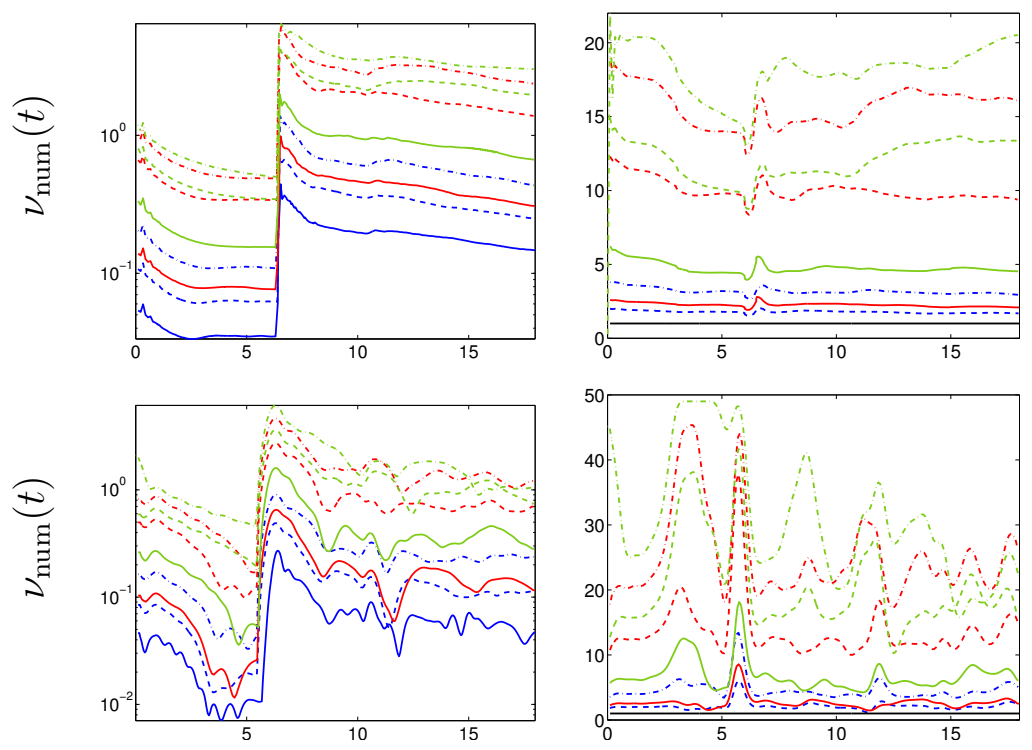

$t$

$t$

Fig. 25. Comparison of the time-evolution of the numerical viscosity $\nu_{\text {num }}(t)$ obtained using the turbulent kinetic energy production/dissipation rate of Eq. (18) (top, left) and Eq. (17) (bottom, left) when the order and resolution are varied. The numerical viscosities normalized by that from the WENO9F simulation are also shown (right column). See Fig. 7 for the legend.

in the flow, while the latter is associated directly with turbulent quantities. The WENO9F simulation attains the largest Reynolds numbers (based on both definitions), which is consistent with the fact that this simulation has the least intrinsic numerical dissipation. The temporal decay of the Reynolds number $\operatorname{Re}_{\ell}$ is consistent with a decaying turbulent flow following the reshock of the mixing layer, in this case with the decay mechanism provided by the numerical dissipation.

\subsection{The numerical viscosity}

Dimensionally, an effective numerical viscosity (dissipation) can be computed from the numerical Kolmogorov length scale and the turbulent kinetic energy production/dissipation rate as

$$
\nu_{\text {num }}(t)=\ell_{d}^{4 / 3} \tilde{\epsilon^{\prime \prime}}(t)^{1 / 3}
$$

This presupposes an analogy between molecular dissipation and numerical dissipation. Two numerical viscosities can be computed using the turbulent kinetic energy production/dissipation rate given by Eqs. (17) and (18).

Figure 24 shows the time-evolution of the numerical viscosities $\nu_{\text {num }}(t)$ as 
the order and resolution are varied, using $\tilde{\epsilon^{\prime \prime}}(t)$ given by Eqs. (17) and (18). Increasing the order and resolution corresponds to simulations with decreasing numerical viscosity (i.e., dissipation). The numerical viscosity increases at reshock, corresponding to additional deposition of energy in the mixing layer. This is followed by a decrease at late times. The numerical viscosity computed using the turbulent kinetic energy production/dissipation rate of Eq. (17) shows additional noise, due to the noisy values of $\tilde{\epsilon}^{\prime \prime}(t)$ (also see Fig. 20). However, the qualitative results are similar. The extreme cases correspond to a difference of one order of magnitude or greater in the value of $\nu_{\text {num }}(t)$ at any given time: the ratios of the numerical viscosities to that from the WENO9F simulation indicate that increasing the order from fifth to ninth constitutes a $\approx 2.5$-fold decrease in the numerical viscosity, while doubling the resolution results in only a $\approx 1.5$-fold decrease. Similar trends are found when the order is changed from third to fifth and the resolution is doubled.

In summary, the sensitivity exhibited by the fields, mixing layer width, circulation, mixing profiles, chemical products, mixing fractions, energy spectra, statistics and probability distribution functions considered in this paper can largely be explained by the different implicit numerical dissipation associated with each given order and resolution. The numerical viscosity computed from the simulation data confirms that the higher-order higher-resolution simulations correspond to lower values of numerical dissipation. It was also shown that doubling the resolution corresponds to an $\approx 1.5$-fold decrease in the numerical viscosity. By contrast, approximately doubling the order corresponds to an $\approx 2.5$-fold decrease in numerical viscosity. Therefore, for the complex hydrodynamic flow considered here, approximately doubling the order yields a larger decrease in the numerical dissipation than that given by doubling the resolution.

\section{Discussion and conclusions}

Simulations of the two-dimensional reshocked single-mode Richtmyer-Meshkov instability were performed using the WENO method with third-, fifth- and ninth-order flux reconstruction and uniform spatial grid resolutions of 128, 256 and 512 points per initial perturbation wavelength. The dependence of the following quantities on the order and resolution was investigated: (1) the density, vorticity, simulated density Schlieren, and the baroclinic vorticity production fields; (2) the mixing layer width; (3) the circulation; (4) mixing profiles; (5) chemical products and mixing fractions; (6) energy spectra; (7) statistics; (8) probability distribution functions (PDFs); (9) the turbulent kinetic energy production/dissipation rate; (10) the turbulent enstrophy production/dissipation rate; (11) numerical Reynolds numbers, and; (12) the numerical viscosity. To our knowledge, this is the first systematic investiga- 
tion of the predictions of the WENO method applied to such quantities as a function of order and resolution in the case of the reshocked RichtmyerMeshkov instability or any other flow. This broad range of quantities includes large-scale dominated quantities (e.g., the mixing layer width), primitive fields (e.g., the density), derived fields (e.g., the vorticity and the density and pressure gradients), spatially-averaged quantities (e.g., the mixing profiles), quantities characterizing the distribution of fluctuations across scales (spectra), global quantities (statistics), and quantities characterizing the range of values (PDFs).

The density, vorticity, baroclinic vorticity production, and simulated density Schlieren fields were compared before and after reshock. Prior to reshock, the density fields from higher-order higher-resolution simulations exhibited additional finer-scale structure in the roll-up. The vorticity fields from higherorder higher-resolution simulations exhibited formation of strong localized cores surrounded by a bilayer with vorticity of opposite sign, followed by late-time fragmentation of the roll-up. Such fragmentation does not occur in the lower-order lower-resolution simulations. At reshock, the simulated density Schlieren fields were used to visualize the waves. The Schlierens from lower-order lower-resolution simulations exhibited thicker transmitted and reflected waves, while the higher-order higher-resolution simulations exhibited sharper features, consistent with numerical dissipation smears the waves. The densities from higher-order higher-resolution simulations exhibited symmetry breaking and the concomitant formation of complex small-scale structures at late times. In contrast, the densities from lower-order lower-resolution simulations exhibited symmetric large-scale structures and vorticities with strong localized cores. The formation of large-scale structures is consistent with the cascade of kinetic energy from small to large scales in two-dimensional flows: this mechanism is more pronounced in the lower-order lower-resolution simulations.

Prior to reshock, the mixing layer widths $h(t)$ were nearly the same among the simulations. However, following reshock and particularly following the arrival of the reflected rarefaction, the widths exhibited larger differences among the simulations. Lower-order lower-resolution simulations have larger numerical diffusion: increased diffusion damps the velocity fluctuations and, in particular, the streamwise fluctuations associated with the growth of the mixing layer along the shock propagation direction. Therefore, increased numerical diffusion is consistent with smaller mixing layer widths. In addition, the formation of small-scale structures was inhibited in simulations with large numerical diffusion, resulting in smaller baroclinic circulation deposition during a waveinterface interaction compared to a higher-order higher-resolution simulation exhibiting much more small-scale structure.

The mixing profiles showed different degrees of sensitivity to the order and 
resolution. Prior to and immediately following reshock, the differences are due to the order and resolution. The differences in the mixing fractions at late times are also due to significant differences in the underlying flow structure. Higher-order higher-resolution simulations exhibited fragmented structured with localized mixed regions, resulting in more pronounced localized peaks in the profiles. Instead, lower-order lower-resolution simulations exhibited more coherent large-scale structures, resulting in fewer peaks spread over a larger portion of the mixing layer. As a result, the mole fraction profile $\bar{X}$ measuring the mass distribution had similar values prior to and immediately following reshock as the order and resolution were increased. By contrast, the averaged product mole fraction $\bar{X}_{p}$ exhibited differences for all times.

The chemical products and mixing fractions were strongly influenced by the numerical dissipation, which is in turn related to the appearance of smallscale structures within the mixing layer at late times following reshock. Loworder low-resolution simulations with increased numerical dissipation resulted in greater overall numerical mixing as measured by the production mixing fraction $P_{t}$ before and after reshock. High-order high-resolution simulations with smaller numerical dissipation had smaller values of $P_{t}$. Prior to reshock, low-order low-resolution simulations had larger values of the mixing fraction $\Xi$, consistent with increased mixing due to the larger numerical dissipation. However, following reshock, $\Xi$ was not very sensitive to the order and resolution. Similar mechanisms also explain the behavior of the mixing fraction $\Theta$, which exhibited stronger sensitivity than $\Xi$ to order and resolution.

The energy spectra exhibited different degrees of sensitivity to the order and resolution. These differences are apparent at all times and become larger at late times, after oscillations have been damped. In particular, the turbulent kinetic energy spectrum $E(k, t)$ exhibited little sensitivity to the order and resolution, whereas the turbulent enstrophy spectrum $E_{\omega^{\prime \prime} \omega^{\prime \prime}}(k, t)$ and the baroclinic vorticity production variance spectrum $E_{\mathcal{P P}}(k, t)$ exhibited significantly more sensitivity, with higher-order lower-resolution simulations exhibiting larger values over a broader range of scales. The density variance spectrum $E_{\rho^{\prime} \rho^{\prime}}(k, t)$ also exhibited sensitivity to the order and resolution at large $k$, while the pressure variance spectrum $E_{p^{\prime} p^{\prime}}(k, t)$ exhibited increased sensitivity at both small and large $k$. These results indicate that spectra corresponding to derived quantities (i.e., quantities depending on spatial derivatives such as the vorticity and baroclinic vorticity production) are very sensitive to the order and resolution.

The statistics also exhibited different degrees of sensitivity to order and resolution. These sensitivities are similar to those in the corresponding energy spectra. The turbulent kinetic energy $E(t)$ exhibited small differences both prior to and following reshock, with the difference prior to reshock due to the complex structure observed in the roll-up. A more significant difference was observed in the spanwise turbulent kinetic energy $E_{v^{\prime \prime} v^{\prime \prime}}(t)$ prior to and fol- 
Table 2

\begin{tabular}{|c||c|c|c|}
\hline & Coarse (128) & Medium (256) & Fine (512) \\
\hline \hline Ninth-order & 0.4 & 2.3 & 20.7 \\
Fifth-order & 0.2 & 1.0 & 9.5 \\
Third-order & 0.17 & 0.8 & 7.1 \\
\hline
\end{tabular}

Ratio of CPU times for advancing the simulations by $\Delta t=0.1 \mathrm{~ms}$ compared with the time needed for the fifth-order simulation at medium resolution.

lowing reshock due to the spanwise fluctuations excited by the roll-up and the complex structures in the flow. The turbulent enstrophy $E_{\omega^{\prime \prime} \omega^{\prime \prime}}(t)$ and baroclinic vorticity production variance $E_{\mathcal{P P}}(t)$ exhibited significant variation over all times. The density and pressure variance $E_{\rho^{\prime} \rho^{\prime}}(t)$ and $E_{p^{\prime} p^{\prime}}(t)$ also exhibited variations prior to and following reshock.

When fluctuating fields or gradients of fields are considered, the higher-order higher-resolution simulations exhibited broader PDFs corresponding to the presence of a wider range of field or gradient values (positive or negative). This has important implications for using numerical simulation data for the evaluation of turbulent transport or subgrid-scale models, as lower-order lowerresolution simulations are excessively diffusive and strongly limit the range of numerical values of quantities supported on a given computational grid.

In summary, the findings presented in this paper were largely interpreted in terms of different relative degrees of implicit numerical dissipation associated with each order and resolution. The numerical dissipation was quantitatively estimated by first determining the turbulent kinetic energy production/dissipation rate $\tilde{\epsilon}^{\prime \prime}(t)$. Higher levels of turbulent kinetic energy and enstrophy production/dissipation are associated with higher-order higher-resolution simulations, as the kinetic energy and enstrophy content in these simulations is higher. The numerical viscosity computed using $\tilde{\epsilon}^{\prime \prime}(t)$ and an estimated Kolmogorov dissipation length scale confirmed that higher-order higher-order simulations correspond to lower numerical dissipation. It was shown that doubling the resolution corresponds to an $\approx 1.5$-fold decrease in the numerical viscosity. By contrast, approximately doubling the order corresponds to an $\approx 2.5$-fold decrease in numerical viscosity. This indicates that high-order (at least ninth-order) WENO methods must be used if either explicit molecular dissipation and diffusion, or explicit subgrid-scale or turbulent transport and mixing (i.e., Reynolds-averaged Navier-Stokes) models are included in such simulations. Furthermore, much larger effective numerical Reynolds numbers can be attained in high-order high-resolution simulations.

Finally, a note regarding the relative computational cost of the simulations presented here, which were conducted on the Blue Pacific computer at the Lawrence Livermore National Laboratory. For each simulation, 32 nodes were 
used with a total of 128 processors. The CPU times required to advance the simulation between 12.5 and $12.6 \mathrm{~ms}$ were compared to the time required for the WENO5M simulation. The ratios of the CPU times to that for the WENO5M simulation are presented in Table 2, which shows that increasing the order requires twice the computational time, whereas doubling the resolution requires five to eight times more computational time.

The lower numerical dissipation associated with formally high-order methods, coupled with the consideration of the computational cost suggests that these methods are suited for simulating complex multi-scale flows with shocks. Simulations with lower numerical dissipation preserve additional small-scale structures and, therefore, provide a more complete representation of the flow dynamics. Low-order coarse-grid representations of complex flow physics can be considerably different than high-order representations and, in general, a broad range of quantities should be considered to differentiate between the predictions of different numerical methods. In addition, a comparison of the relative CPU times suggests that the utilization of higher-order methods is more computationally efficient than increasing the grid resolution for the twodimensional Richtmyer-Meshkov instability, i.e., a complex, multi-dimensional flow containing shocks (and other waves) and a wide range of scales. Thus, the use of formally high-order methods can lead to a significant advantage in multi-dimensional simulations of such flows. While definitive experimental data corresponding to the quantities considered in this paper is required to determine which simulation order and resolution are optimal for a given flow, it is likely that higher-order higher-resolution simulations provide data with higher fidelity. The analysis presented in this paper is currently being applied to a three-dimensional reshocked multi-mode Richtmyer-Meshkov instabilityinduced flow.

\section{Acknowledgments}

The authors thank Dr. Daniel I. Meiron at the California Institute of Technology for useful discussions. Marco Latini gratefully acknowledges support from the Air Force Office of Scientific Research (AFOSR) through the National Defense Science and Engineering Graduate (NDSEG) Fellowship and the Caltech Accelerated Strategic Computing (ASC) Center. Wai Sun Don gratefully acknowledges support of this work by the DOE under contract number DEFG02-98ER25346 and by the AFOSR under contract number FA9550-05-10123. This work was also performed under the auspices of the U.S. Department of Energy by the University of California, Lawrence Livermore National Laboratory under contract No. W-7405-Eng-48. 


\section{References}

[1] D. Balsara, C.-W. Shu, Monotonicity preserving weighted essentially nonoscillatory schemes with increasingly high order of accuracy, J. Comput. Phys. 160 (2000) 405-452.

[2] M. Latini, O. Schilling, W. S. Don, High-resolution simulations and modeling of reshocked single-mode Richtmyer-Meshkov instability. I. Comparison to experimental data and to amplitude growth model predictions, Phys. FluidsSubmitted.

[3] B. D. Collins, J. W. Jacobs, PLIF flow visualization and measurements of the Richtmyer-Meshkov instability of an air $/ \mathrm{SF}_{6}$ interface, J. Fluid Mech. 464 (2002) 113-136.

[4] O. Schilling, M. Latini, W. S. Don, High-resolution simulations and modeling of reshocked single-mode Richtmyer-Meshkov instability. II. Physics of reshock and mixing, Phys. FluidsSubmitted.

[5] G. Tryggvason, Numerical simulations of the Rayleigh-Taylor instability, J. Comput. Phys. 75 (1988) 253-282.

[6] X. He, S. Chen, R. Zhang, A lattice Boltzmann scheme for incompressible multiphase flow and its application in simulation of Rayleigh-Taylor instability, J. Comput. Phys. 152 (1999) 642-663.

[7] G. Dimonte, D. L. Youngs, A. Dimits, S. Weber, M. Marinak, S. Wunsch, C. Garasi, A. Robinson, M. J. Andrews, P. Ramaprabhu, A. C. Calder, B. Fryxell, J. Biello, L. Dursi, P. MacNeice, K. Olson, P. Ricker, R. Rosner, F. Timmes, H. Tufo, Y.-N. Young, M. Zingale, A comparative study of the turbulent Rayleigh-Taylor instability using high-resolution three-dimensional numerical simulations: The Alpha-Group collaboration, Phys. Fluids 16 (2004) 1668-1693.

[8] R. L. Holmes, J. W. Grove, D. H. Sharp, Numerical investigation of RichtmyerMeshkov instability using front-tracking, J. Fluid Mech. 301 (1995) 51-64.

[9] R. L. Holmes, G. Dimonte, B. Fryxell, M. L. Gittings, J. W. Grove, M. Schneider, D. H. Sharp, A. L. Velikovich, R. P. Weaver, Q. Zhang, RichtmyerMeshkov instability growth: experiment, simulation and theory, J. Fluid Mech. 389 (1999) 55-77.

[10] R. H. Cohen, W. P. Dannevik, A. M. Dimits, D. E. Eliason, A. A. Mirin, Y. Zhou, D. H. Porter, P. R. Woodward, Three-dimensional simulation of a Richtmyer-Meshkov instability with a two-scale initial perturbation, Phys. Fluids 14 (2002) 3692-3709.

[11] J. Shi, Y.-T. Zhang, C.-W. Shu, Resolution of high order WENO schemes for complicated flow structures, J. Comput. Phys. 186 (2003) 690-696. 
[12] A. W. Cook, W. H. Cabot, J. A. Greenough, A High-Order Method for ShockInduced Mixing, Tech. Rep. UCRL-JC-144109, Lawrence Livermore National Laboratory (2001).

[13] J. P. Boris, F. F. Grinstein, E. S. Oran, R. L. Kolbe, New insights into large eddy simulation, Fluid Dyn. Res. 10 (1992) 199-228.

[14] D. Drikakis, Embedded turbulence model in numerical methods for hyperbolic conservation laws, Int. J. Num. Meth. Fluids 39 (2002) 763-781.

[15] D. Drikakis, Advances in turbulent flow computations using high-resolution methods, Prog. Aero. Sci. 39 (2003) 405-424.

[16] D. Drikakis, F. Grinstein, D. Youngs, On the computation of instabilities and symmetry-breaking in fluid mechanics, Prog. Aero. Sci. 41 (2005) 609-641.

[17] H. O. Kreiss, J. Oliger, Comparison of accurate methods for integration of hyperbolic equations, Tellus 24 (1972) 199-215.

[18] L. Jameson, High order schemes for resolving waves: number of points per wavelength, J. Sci. Comput. 15 (2000) 417-439.

[19] B. Costa, W. S. Don, Hybrid central-WENO finite difference methods for conservation laws, J. Comput. App. Math.Accepted.

[20] D. J. Hill, D. I. Pullin, Hybrid tuned center-difference-WENO method for large eddy simulations in the presence of strong shocks, J. Comput. Phys. 194 (2004) 435-450.

[21] S. Pirozzoli, Conservative hybrid compact-WENO scheme for shock-turbulence interaction, J. Comput. Phys. 178 (2002) 81-117.

[22] Y. X. Ren, M. Liu, H. X. Zhang, A characteristic-wise hybrid compact-WENO scheme for solving hyperbolic conservation laws, J. Comput. Phys. 192 (2003) $365-386$.

[23] D. Kim, J. H. Kwon, A high-order accurate hybrid scheme using a central flux scheme and a WENO scheme for compressible flowfield analysis, J. Comput. Phys. 210 (2005) 554-583.

[24] M. R. Meloon, Models of Richtmyer-Meshkov instability in continuously stratified fluids, Ph.D. thesis, California Institute of Technology (1998).

[25] N. J. Zabusky, A. D. Kotelnikov, Y. Gulak, G. Peng, Amplitude growth rate of a Richtmyer-Meshkov unstable two-dimensional interface to intermediate times, J. Fluid Mech. 475 (2003) 147-162.

[26] G. Peng, N. J. Zabusky, S. Zhang, Vortex-accelerated secondary baroclinic vorticity deposition and late-intermediate time dynamics of a two-dimensional Richtmyer-Meshkov interface, Phys. Fluids 15 (2003) 3730-3744.

[27] J. W. Jacobs, The dynamics of shock accelerated light and heavy gas cylinders, Phys. Fluids A 5 (1993) 2239-2247. 
[28] C. E. Niederhaus, Experiments on the Richtmyer-Meshkov Instability of Incompressible Fluids, Ph.D. thesis, University of Arizona (2000).

[29] J. W. Jacobs, V. V. Krivets, Experiments on the late-time development of single-mode richtmyer-meshkov instability, Phys. Fluids 17 (2005) 034104-1034105-10.

[30] A. Marquina, P. Mulet, A flux-split algorithm applied to conservative models for multicomponent compressible flows, J. Comput. Phys 185 (2003) 120-138.

[31] K. O. Mikaelian, Growth rate of the Richtmyer-Meshkov instability at shocked interfaces, Phys. Rev. Lett. 71 (1993) 2903-2906.

[32] A. W. Cook, P. E. Dimotakis, Transition stages of Rayleigh-Taylor instability between miscible fluids, J. Fluid Mech. 443 (2001) 69-99, corrigendum, J. Fluid Mech. 457 (2002), 410.

[33] D. L. Youngs, Numerical simulation of mixing by Rayleigh-Taylor and Richtmyer-Meshkov instabilities, Laser Part. Beams 12 (1994) 725-750.

[34] D. L. Youngs, Three-dimensional numerical simulations of turbulent mixing by Rayleigh-Taylor instability, Phys. Fluids A 3 (1991) 1312-1320.

[35] M. M. Koochesfahani, P. E. Dimotakis, Mixing and chemical reactions in a turbulent liquid mixing layer, J. Fluid Mech. 170 (1986) 83-112.

[36] J. A. Domaradzki, S. Radhakrishnan, Effective eddy viscosities in implicit modeling of decaying high Reynolds number turbulence with and without rotation, Fluid Dyn. Res. 36 (2005) 385-406.

[37] D. C. Wilcox, Turbulence Modeling for CFD, DCW Industries Inc., La Cañada, CA, 1998.

[38] A. R. Miles, B. Blue, M. J. Edwards, J. A. Greenough, J. F. Hansen, H. F. Robey, Transition to turbulence and effect of initial conditions on threedimensional compressible mixing in planar blast-wave-driven systems, Phys. Plasmas 12 (2005) 056317-1-056317-10.

[39] G. K. Batchelor, The Theory of Homogeneous Turbulence, Cambridge Monographs on Mechanics and Applied Mathematics, Cambridge University Press, New York, 1953.

[40] P. E. Dimotakis, The mixing transition in turbulent flows, J. Fluid Mech. 409 (2000) 69-98.

[41] R. H. Kraichnan, D. Montgomery, Two-dimensional turbulence, Rep. Prog. Phys. 43 (1980) 547-619. 